\title{
PD-L1 in tumor microenvironment mediates resistance to oncolytic immunotherapy
}

\author{
Dmitriy Zamarin, ${ }^{1,2,3,4,5}$ Jacob M. Ricca, ${ }^{3,4}$ Svetlana Sadekova, ${ }^{6}$ Anton Oseledchyk, ${ }^{3,4}$ Ying Yu, ${ }^{6}$ Wendy M. Blumenschein, ${ }^{6}$ \\ Jerelyn Wong, ${ }^{6}$ Mathieu Gigoux, ${ }^{3,4}$ Taha Merghoub, ${ }^{2,3,4,5}$ and Jedd D. Wolchok ${ }^{1,2,3,4,5}$ \\ 'Department of Medicine, Memorial Sloan Kettering Cancer Center (MSKCC), New York, New York, USA. 'Weill Cornell Medical College, New York, New York, USA. ${ }^{3}$ Ludwig Collaborative Laboratory, \\ ${ }^{4}$ Swim Across America Laboratory, and ${ }^{5}$ Parker Institute for Cancer Immunotherapy, MSKCC, New York, New York, USA. ${ }^{6}$ Merck Research Labs (MRL), Palo Alto, California, USA.
}

\begin{abstract}
Intralesional therapy with oncolytic viruses (OVs) leads to the activation of local and systemic immune pathways, which may present targets for further combinatorial therapies. Here, we used human tumor histocultures as well as syngeneic tumor models treated with Newcastle disease virus (NDV) to identify a range of immune targets upregulated with OV treatment. Despite tumor infiltration of effector T lymphocytes in response to NDV, there was ongoing inhibition through programmed death ligand 1 (PD-L1), acting as a mechanism of early and late adaptive immune resistance to the type I IFN response and T cell infiltration, respectively. Systemic therapeutic targeting of programmed cell death receptor 1 (PD-1) or PD-L1 in combination with intratumoral NDV resulted in the rejection of both treated and distant tumors. These findings have implications for the timing of PD-1/PD-L1 blockade in conjunction with OV therapy and highlight the importance of understanding the adaptive mechanisms of immune resistance to specific OVs for the rational design of combinatorial approaches using these agents.
\end{abstract}

\section{Introduction}

In the past 60 years, multiple studies have evaluated the antitumor potential of oncolytic viruses (OVs), and while promising activity has been demonstrated in a variety of animal models, clinical results have not been as impressive. The major limitations of OVs are their poor delivery to metastatic cancer sites with systemic administration and rapid development of neutralizing antibodies. Nevertheless, in the few patients who responded to OVs, the observed clinical benefit was often durable, even after completion of therapy (1). These studies implicate the role of the antitumor immune response in the observed therapeutic effect of OVs and warrant further exploration of OVs as immunotherapeutic, rather than simply lytic, agents. The results using intratumorally administered OVs have been more impressive. Recently, a phase III trial with an oncolytic herpes simplex virus (HSV) expressing granulocyte macrophage colony-stimulating factor (GM-CSF) (talimogene laherparepvec or $\mathrm{T}-\mathrm{vec}$ ) showed a durable benefit from intratumoral therapy, with responses observed in both injected and noninjected lesions $(2,3)$, leading to approval of T-vec by the FDA for the treatment of advanced melanoma. These responses, however, were not universal and, in particular, were rare in patients with more advanced, visceral disease, which suggests that

\section{Related Commentary: p. 1258}

Authorship note: TM and JDW contributed equally to this work.

Conflict of interest: DZ and JDW are inventors on a patent concerning the uses of recombinant Newcastle disease virus for cancer therapy (US 20160015760). SS, YY, JW, and WMB are employees of Merck \& Co. Inc.

Submitted: October 12, 2017; Accepted: December 28, 2017.

Reference information: J Clin Invest. 2018;128(4):1413-1428.

https://doi.org/10.1172/JCI98047. tumor-exerted immunosuppressive mechanisms may be responsible for this lack of efficacy (3).

Newcastle disease virus (NDV) is an avian paramyxovirus that selectively replicates in cells with deficiency in apoptotic and innate immune responses (4). We have recently demonstrated that intratumoral therapy with nonpathogenic NDV results in increased immune infiltration into distant tumors not affected by the virus directly (5), mirroring the results observed with $\mathrm{T}$-vec in patients. Combination of intratumoral NDV with systemic CTLA-4 blockade resulted in the efficient rejection of both virus-injected and distant tumors (5). Intratumoral modulation of the ICOS pathway by NDV led to further enhancement of local and abscopal immune effects, suggesting that targeting immune pathways on both local and systemic levels could optimize the therapeutic efficacy of such approaches (6). Recent clinical trials involving patients with melanoma that evaluated intratumoral $\mathrm{T}$-vec with systemic cytotoxic $\mathrm{T}$ lymphocyte-associated protein 4 (CTLA-4) or programmed cell death receptor 1 (PD-1) blockade showed very promising response rates, further highlighting the therapeutic potential of OVs when used in combination with systemic immunomodulation (7-9).

Given these findings, in the current study we sought to explore the mechanisms underlying the rationale for OV therapy and systemic immunomodulation. In particular, the type I IFN pathway has emerged as an important regulator of the antitumor immune response, with several studies demonstrating its role in antigen presentation by $\mathrm{CD}^{+}$DCs (10-12). NDV has been previously demonstrated to be a strong activator of type I IFN and DC maturation (13), and therapeutic efficacy of NDV in combination with CTLA-4 blockade was completely abrogated in type I IFN receptorKO (IFNAR-KO) mice, highlighting an indispensable role for the type I IFN pathway in OV-mediated antitumor immunity (5). 
Both OVs and the OV-activated type I IFN pathway can exert a variety of pleiotropic effects on immune and nonimmune cells, activating resistance to the immune system on both local and abscopal levels. In the current study, using transcriptional profiling of human tumor histocultures infected ex vivo with NDV as a model OV as well as syngeneic mouse tumor models bearing virus-treated and distant tumors, we identified a range of immune-inhibitory genes that were upregulated at the NDVinjected and distant sites and specifically focused on the PD-1/ programmed death ligand 1 (PD-1/PD-L1) pathway, given the clinical advances in anticancer therapies targeting these proteins. We found that while NDV therapy shifted the balance from exhausted to the effector $\mathrm{T}$ cell phenotype in both virus-injected and distant tumors, it was not sufficient for complete tumor rejection. We further show that infection with NDV led to the upregulation of PD-L1 in tumor cells and tumor-infiltrating immune cells as an immediate early response to type I IFN and as a late adaptive mechanism of immune resistance to the increased immune cell infiltration into the tumor. Combination therapy of intratumorally administered NDV and systemic PD-1 or PD-L1 blockade resulted in a marked enhancement of the antitumor immune effect, leading to rejection of the OV-treated and distant, noninfected tumors. These findings highlight an important mechanism of resistance to the efficacy of NDV and possibly other OVs and provide a rationale for the consideration of the timing of combinations of OVs and PD-1/PD-L1 blockade in clinical trials.

\section{Results}

Locoregional NDV therapy produces local and abscopal immune effects. To model the immune effects of intratumoral NDV therapy, cultured tumor explants from several tumor types obtained from patients were infected ex vivo with NDV and analyzed for transcriptional changes 24 hours after infection using quantitative real-time PCR (RT-PCR). Infection with NDV resulted in strong upregulation of a number of immune-related genes, including type I IFN-related transcripts and transcripts encoding a range of chemokines, many of which were previously associated with a response to PD-1 blockade $(14,15)$ (Figure 1A; Supplemental Figure $1 \mathrm{~A}$; supplemental material available online with this article; https://doi.org/10.1172/JCI98047DS1). Interestingly, we detected variability across the samples, several of which showed an almost complete lack of response to NDV. This appeared not to be secondary to differences in infectivity with NDV, as both responsive and nonresponsive tumor samples had similar levels of NDV RNA at 24 hours (Supplemental Figure 1, B and C). We characterized the tumor samples according to the presence of transcripts defining specific myeloid and lymphoid cellular subsets and found that the presence of transcripts associated with B cell infiltration was associated with a lack of response to NDV (Figure 1B). Previous studies have demonstrated that tumor-infiltrating $\mathrm{B}$ cells can promote tumor growth through the production of cytokines that alter innate signaling pathways such as IKK $\alpha$ in tumor cells (16-18). While there were no baseline differences in the expression of genes involved in innate immune recognition, we observed a defective upregulation of retinoic acid-inducible gene I (RIGI) expression in response to NDV in nonresponsive tumor samples (Supplemental Figure 1D). Given that the RIG-I pathway serves as a major mechanism of
NDV sensing (13), it is possible that defective upregulation of RIG-I dampens downstream immune effects or is simply a marker of an overall defect in innate immune transcriptional activation. It is thus likely that the poor responses to NDV observed in select tumors were either a result of tumor cell-intrinsic defects in innate signaling or of defects induced by the presence of specific cellular subsets such as B cells in the tumor microenvironment.

To characterize these effects in a mouse tumor model, we used a bilateral flank B16-F10 melanoma model, in which the virus was administered to a single-flank tumor (Figure 1C). Of note, in this model, viral infection remains confined to the injected tumor site, which allows for the evaluation of both local and abscopal immune effects (5). Analysis of both treated and distant tumors revealed a marked increase in the infiltrating immune cells of both innate and adaptive lineages (Figure 1D). Notably, there was a prominent increase in the number of infiltrating $\mathrm{CD}^{+}$and conventional $\mathrm{CD}^{+}{ }^{+} \mathrm{FoxP}^{-} \mathrm{T}$ cells (Tcon), with a small, albeit statistically significant, increase in Tregs (Figure 1D). Despite these findings, while intratumoral injection of NDV resulted in significantly delayed growth of both virus-injected and distant tumors (Figure 1E), there was eventual tumors outgrowth that resulted in a modest but statistically significant prolongation of overall survival (Figure $1 \mathrm{E})$. These findings highlight the notion that the NDV-mediated induction of favorable inflammatory changes in the microenvironment of virus-treated and distant tumors is not sufficient to drive complete tumor rejection, implying that potential inhibitory mechanisms may be dampening the immune response.

A broad analysis of gene expression in treated and distant tumors from NDV-treated animals using the NanoString platform revealed the upregulation of multiple immune-related genes related to both innate and adaptive immune responses, with the strongest effects seen in the virus-injected tumors (Figure 2A). Notably, we observed increased expression of a range of immune-inhibitory genes, a number of which are currently being explored as clinical targets for cancer immunotherapy (Figure 2A). While most of these targets support a rationale for further exploration within the context of NDV therapy, we chose to specifically focus on the PD-1/PD-L1 pathway, given the clinical advances in anticancer therapies targeting these proteins and their role in $\mathrm{T}$ cell exhaustion and promotion of chronic viral infections with viruses like HIV, HBV, and HCV, as well as the promising early clinical data on the use of an OV in combination with systemic PD-1 blockade to treat melanoma $(8,19)$.

$\mathrm{T}$ cell exhaustion is associated with chronic antigen stimulation in the context of tumor or chronic viral infection and is characterized by weak effector function, which is in part restrained by the inhibitory activity of the PD-1 receptor (20). At the transcriptional level, $T$ cell exhaustion has been shown to be associated with loss of Tbet $^{+}$precursors, which are necessary for the production of the EOMES $^{+} \mathrm{CD}^{+} \mathrm{T}$ cells that control viral infection (21). We initially sought to determine whether the tumor-infiltrating lymphocyte (TIL) population accumulating in response to NDV therapy had markers known to be associated with $\mathrm{T}$ cell dysfunction, such as PD-1 and LAG3. While there was an increase in PDCD1 and LAG3 expression in the NDV-treated tumors, expression of these genes did not change after treatment in the distant tumors (Figure 2A). Plotting normalized PDCD1 and LAG3 expression levels against 
A

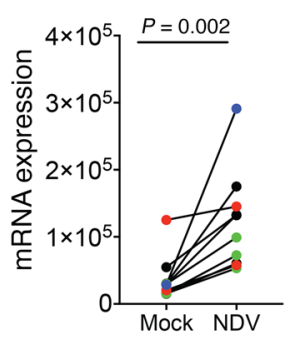

MX2

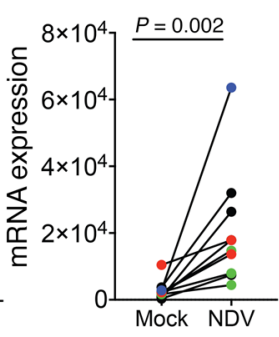

IFIT2

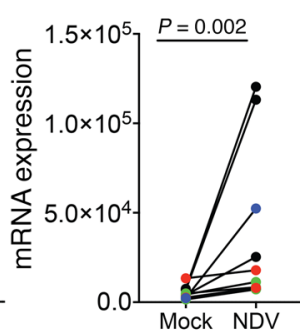

CXCL9

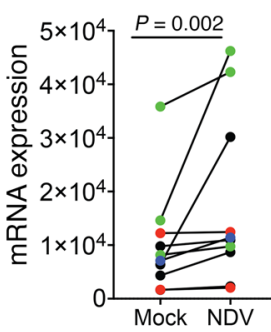

CXCL11

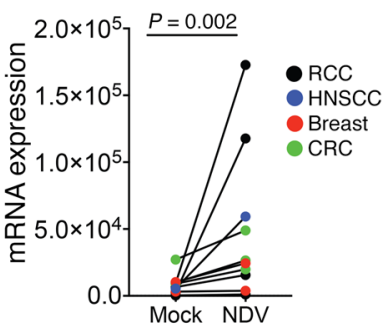

B
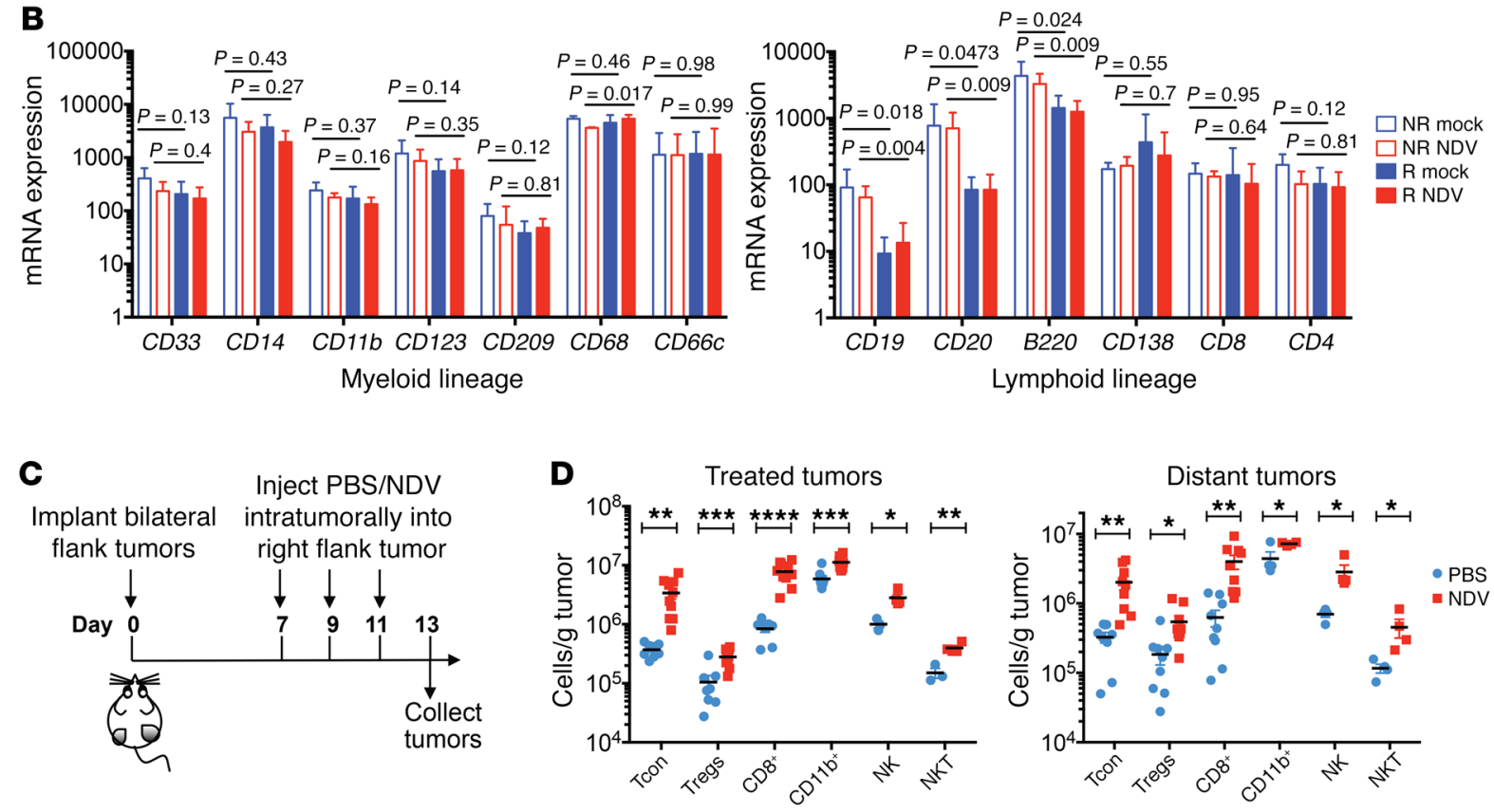

E
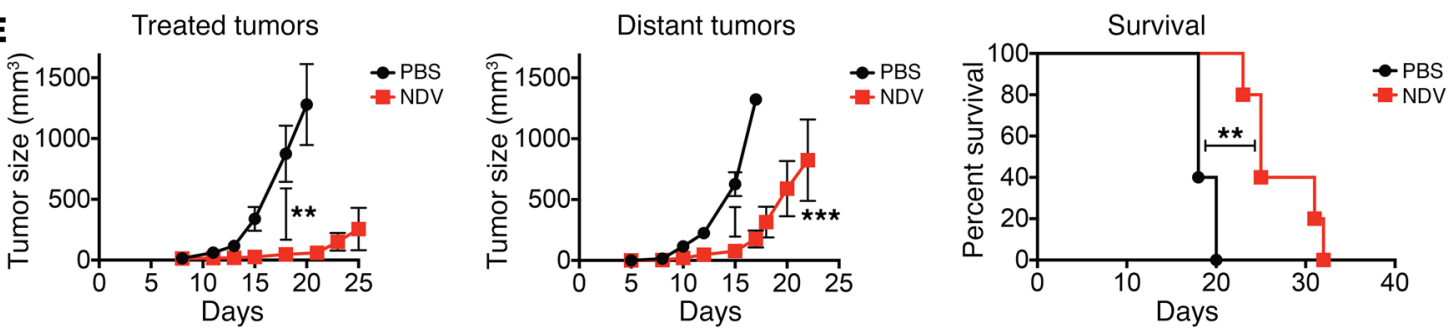

Figure 1. Local and abscopal effects of intratumoral NDV therapy. (A) RCC, CRC, breast cancer, and HNSCC tumor specimens were treated with NDV for 24 hours. Expression of representative type I IFN-related genes and chemokine genes in tumors was determined by real-time quantitative PCR. (B) Expression of myeloid and lymphoid lineage markers by real-time quantitative PCR in the NDV-responding (R) and nonresponding (NR) samples. Data represent 7 responding and 3 nonresponding tumors (see Figure $1 \mathrm{~A}$ and Supplemental Figure 1). (C) Animals bearing bilateral flank B16-F10 tumors were treated with 3 injections of NDV administered into the right-flank tumor. IT, intratumorally. (D) Immune infiltration into the treated and distant tumors was determined by flow cytometry. (E) Growth of the treated and distant tumors and overall survival. (A and B) Each tumor specimen represents an individual experiment. (C-E) Results are representative of 2 independent experiments with 5 to 10 animals per group, and data represent the mean \pm SEM. Data were analyzed using the Wilcoxon matched-pairs, signed-rank test (A), Student's $t$ test for individual comparisons (B and $\mathbf{D}$, and $\mathbf{E}, 2$ left panels), and log-rank test (E, right panel). ${ }^{*} P<0.05,{ }^{* *} P<0.01,{ }^{* * *} P<0.001$, and ${ }^{* * *} P<0.0001$. Tcon, CD4 ${ }^{+}$FoxP3 $^{-}$; Treg, CD4 $^{+}$FoxP3 $^{+}$.

Thet expression revealed a strong correlation between Tbet and $L A G 3$ and Tbet and PDCD1 in NDV-treated animals (Figure 2, B and C). In both treated and distant tumors, NDV treatment resulted in higher levels of Tbet expression in relation to PDCD1 and LAG3, with the most dramatic increase in Tbet expression seen in the treated tumors, in which complete tumor rejections were seen (Figure 1D and Figure 2, B and C). These findings are suggestive of a shift to the effector $\mathrm{T}$ cell phenotype in the treated and, to a lesser extent, distant tumors. In support of this hypothesis, we observed a preferential increase in the granzyme $\mathrm{B}^{+} \mathrm{PD}-\mathrm{1}^{-} \mathrm{CD} 8^{+}$ $\left(\mathrm{GrB}^{+} \mathrm{PD}-\mathrm{1}^{-} \mathrm{CD} 8^{+}\right)$lymphocyte population in response to NDV therapy (Figure 2, D and E), and an overall upregulation of activation (ICOS) and proliferation (Ki-67) markers in the CD8 ${ }^{+}$and Tcon subsets in distant tumors (Figure 2F). These results imply 
A
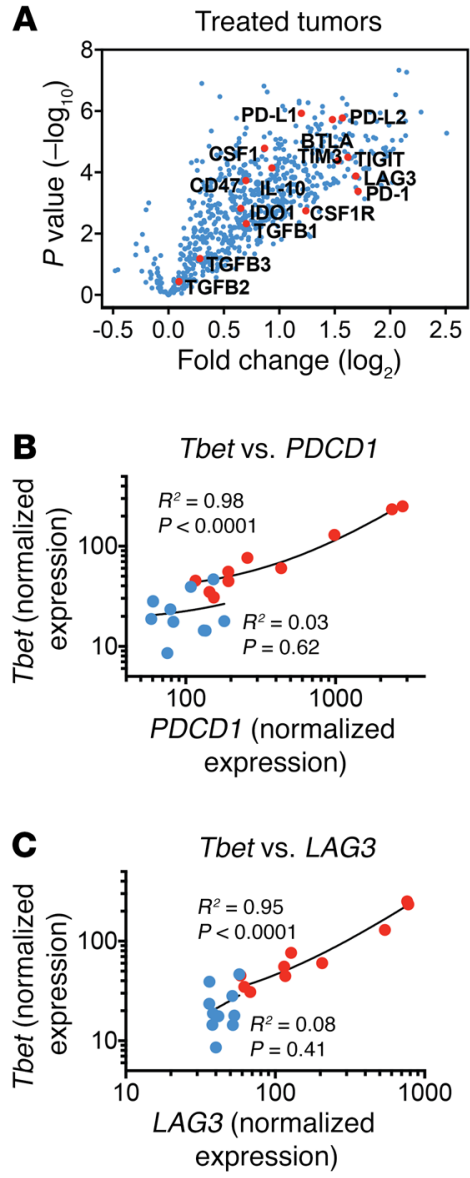

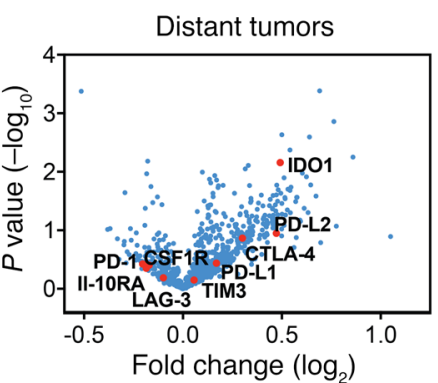

Tbet vs. PDCD1
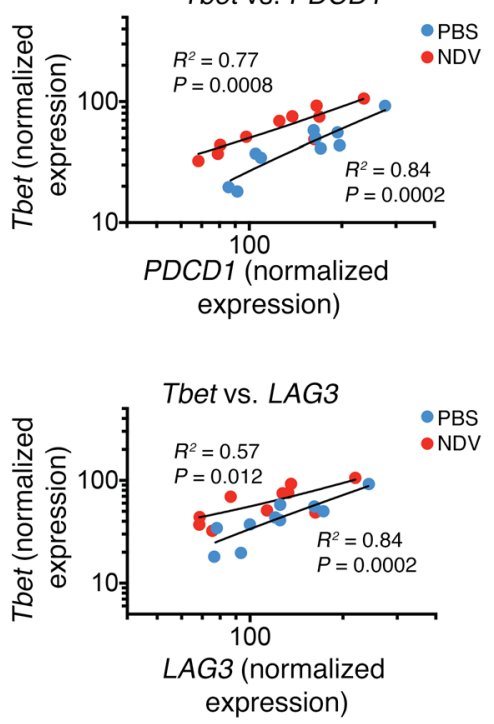
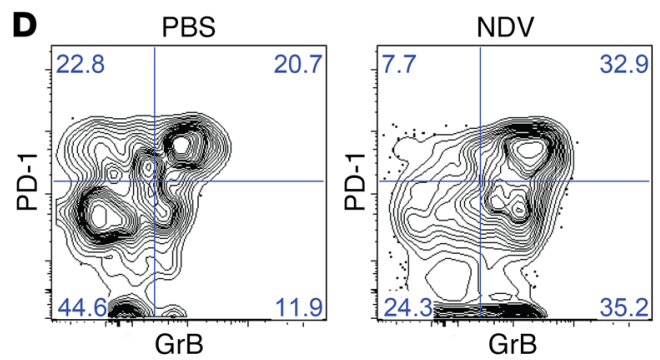

$\mathbf{E}$
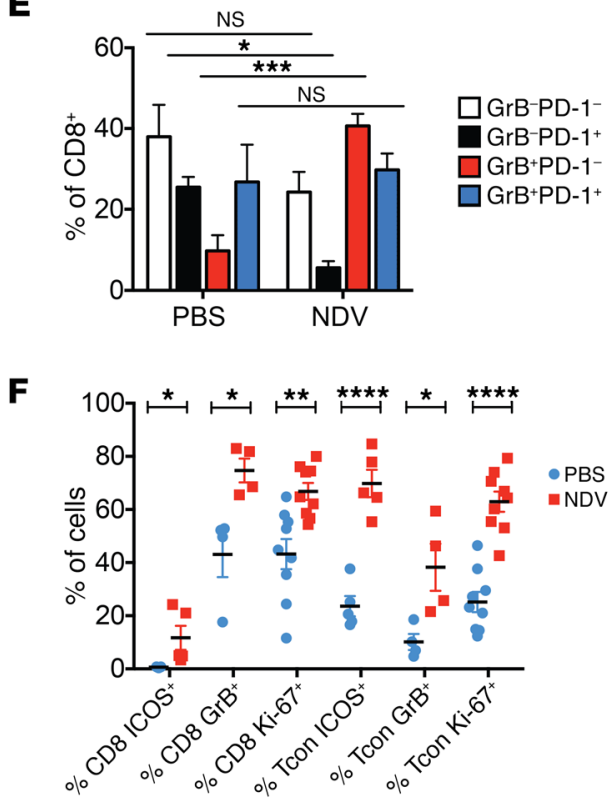

Figure 2. NDV upregulates immune-inhibitory pathways in tumors. (A) Gene expression profiling of the treated and distant tumors analyzed on the NanoString platform. (B and C) Correlation of expression of Tbet versus PDCD1 (B) and Tbet versus LAG3 (C) in the treated (left) and distant (right) tumors, as determined by NanoString. (D and E) Expansion of GrB+PD-1- lymphocytes in response to NDV therapy in distant tumors. (D) Representative flow cytometric plots. (E) Grouped plot of all samples. (F) Expression of activation (ICOS), lytic (GrB'), and proliferation (Ki-67) markers by the CD8 ${ }^{+}$and Tcon lymphocytes from distant tumors as determined by flow cytometry. Results are representative of 2 independent experiments, with 5 to 10 animals per group, and data represent the mean \pm SEM. Data were analyzed using the NanoString Advanced Analysis module for differential expression with the Benjamini-Yekutieli $P$ value adjustment method (A), Pearson's correlation (B and C), 1-way ANOVA with multiple comparisons (E), and Student's $t$ test for individual comparisons (F). ${ }^{*} P<0.05,{ }^{* *} P<0.01,{ }^{* *} P<0.001$, and ${ }^{* * *} P<0.0001$.

that, while on the T cell-intrinsic level NDV therapy results in a balance shift toward the activated rather than exhausted phenotype in distant tumors, this shift is insufficient to drive complete tumor rejection. Given these findings, we sought to determine whether modulation of the PD-1 pathway on the extrinsic level through PD-L1 expression could be a mechanism that inhibits the activation of $\mathrm{T}$ cells by NDV therapy.

The dynamics of NDV-induced PD-L1 upregulation in treated and distant tumors are differentially regulated by innate and adaptive immune responses. Infection of human tumor histocultures with NDV ex vivo revealed upregulation of CD274 (encoding PD-L1) and PDCD1LG2 (encoding PD-L2) to various levels across several tumor types (Figure 3A). Notably, a marked increase in CD274 and $P D C D 1 L G 2$ expression was also seen in the ex vivo-infected whole blood isolated from patients with cancer and from healthy donors, suggesting that the observed upregulation in the infected tumor tissue could be due at least in part to direct infection of tumorinfiltrating immune cells (Figure 3A). To characterize the immune cellular subsets that showed PD-L1 upregulation in response to
NDV in vivo, animals bearing bilateral B16-F10 tumors were treated intratumorally with NDV expressing GFP (NDV-GFP), and the treated tumors and distant tumors were collected either 24 hours later or after 3 treatments on day 13 and analyzed for PD-L1 expression by flow cytometry (Figure 3B). Consistent with the findings in human tumor histocultures, 24 hours after treatment, we observed robust PD-L1 upregulation in the virus-treated tumors (Figure 3C), while no changes in PD-L1 were seen in the distant tumors (Figure 3D). In contrast, late in the course of treatment, we detected PD-L1 upregulation in both virus-treated and distant tumors (Figure 3, $\mathrm{E}$ and F). In both NDV-treated and distant tumors, we detected PD-L1 upregulation on both $\mathrm{CD}^{-} 5^{-}$cells (primarily representing the tumor cell fraction) and the $\mathrm{CD} 45^{+}$cell population, including $\mathrm{CD}_{11 \mathrm{~b}^{+}}$myeloid cells, $\mathrm{CD} 8^{+}$lymphocytes, and $\mathrm{CD} 4^{+} \mathrm{FoxP}^{-}{ }^{-} \mathrm{Tcon}$ cells and Tregs (Figure 3, E and F, and Supplemental Figure 3). Confirming the flow cytometric data, microscopy revealed a robust upregulation of PD-L1 protein expression in response to NDV treatment in distant tumors during the late time point, with PD-L1 ${ }^{+}$cells distributed throughout the tumor (Figure 3G). 

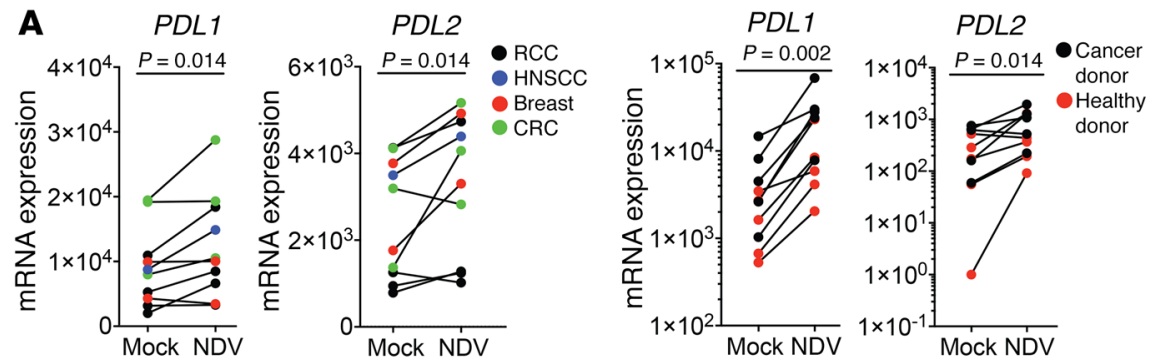

B

Implant bilateral

flank tumors

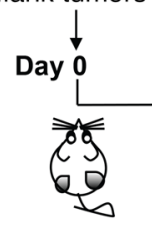

Inject PBS/NDV

intratumorally into

right flank tumor

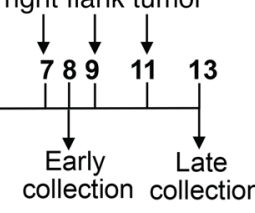

C

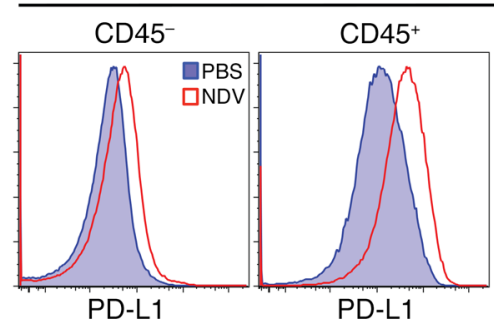

Tumors treated early

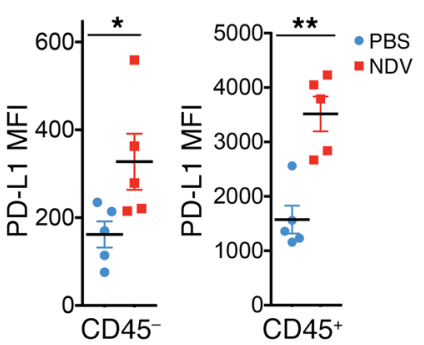

D Distant tumors treated early
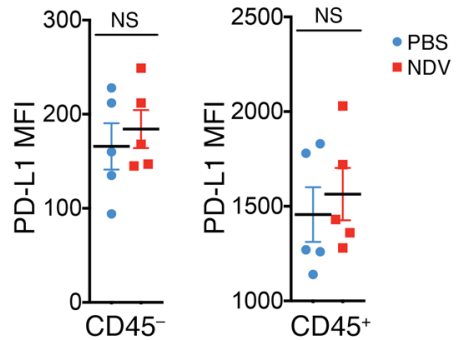

$\mathbf{E}$

Tumors treated late

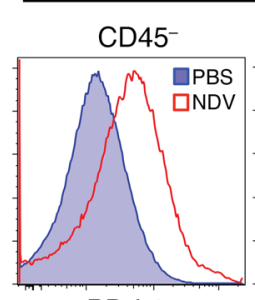

PD-L1

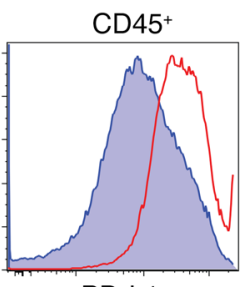

PD-L1

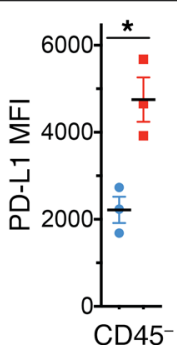

CD45-

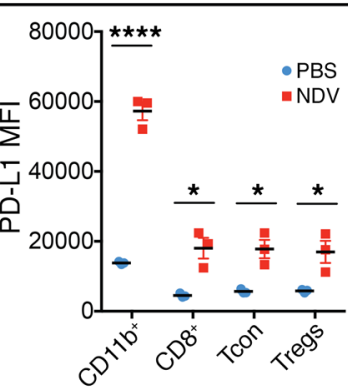

NDV

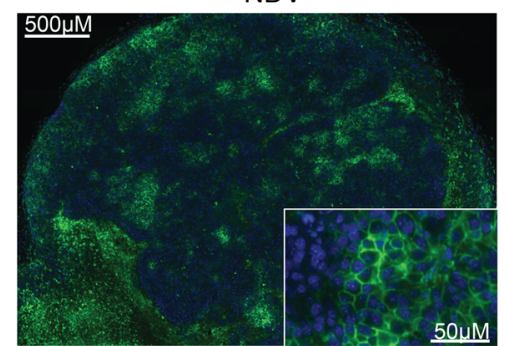

F

Distant tumors treated late
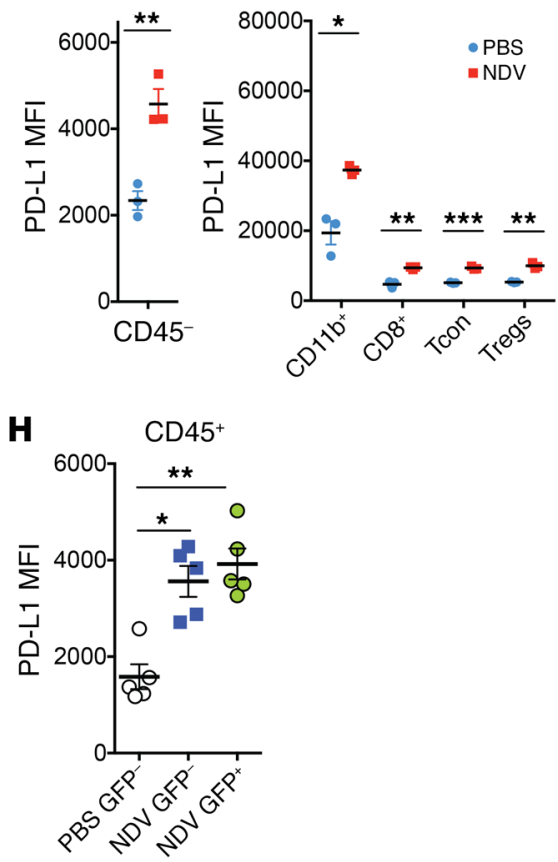

Figure 3. Induction of PD-L1 in NDV-treated and distant tumors. (A) mRNA expression of PDL1 and PDL2 in the cultured NDV-infected tumor specimens $(48 \mathrm{~h})$ and NDV-infected whole blood ( $24 \mathrm{~h}$ ) obtained from healthy donors and from patients with cancer. (B) Mouse treatment schema. Tumors were collected at 24 hours (early) or 6 days (late) after the first treatment. (C and $\mathbf{D})$ Upregulation of PD-L1 on CD45+ and CD45- cells in treated tumors (C) and distant tumors (D) at 24 hours (early). Left: representative flow cytometry histograms; right: quantified PD-L1 MFI. (E and F) Upregulation of PD-L1 on CD45 and CD45- $^{-}$cells in treated tumors (E) and distant tumors (F) on day 6 (late). Shown are representative flow cytometric histograms and quantification of $\mathrm{PD}-\mathrm{L} 1 \mathrm{MFI}$ on $\mathrm{CD}^{-} 5^{-}$cells and on the indicated leukocyte subsets. (C) Expression of PD-L1 in distant tumors on day 6. (H) MFI of PD-L1 expression in GFPand GFP+ $\mathrm{CD}_{4}{ }^{+}$cells isolated from the tumors treated with NDV expressing GFP 24 hours after infection. Scale bars: $500 \mu \mathrm{m}$ and $50 \mu \mathrm{m}$ (enlarged insets). (A) Each specimen represents an individual experiment. (B-H) Results are representative of 3 independent experiments with 3 to 5 animals per group, and data represent the mean \pm SEM. Data were analyzed by a Wilcoxon matched-pairs, signed-rank test $(\mathbf{A})$ and a Student's $t$ test for individual comparisons (C-F and $\mathbf{H}$ ). ${ }^{*} P<0.05,{ }^{* *} P<0.01,{ }^{* *} P<0.001$, and ${ }^{* * *} P<0.0001$. MFI, median fluorescence intensity.

The discordant findings between the early and late time points suggest that different mechanisms drive PD-L1 upregulation early and late in treatment. Data from the infected human histocultures as well as from samples of tumors treated early imply that PD-L1 upregulation in the treated tumors is driven by an early response to viral infection. To determine whether the observed early PD-L1 upregulation in the treated tumors is mediated by direct viral infection of these cell populations, we performed flow cytometry to ana- 
lyze the tumors isolated 24 hours after treatment for GFP expression. We found that GFP expression was detectable in a very small fraction of CD $45^{-}$and CD $45^{+}$cells (Supplemental Figure 4A), with GFP signal predominantly seen in myeloid cells and DCs in the latter cell population (Supplemental Figure 4B). Despite the small fraction of infected cells, we detected PD-L1 upregulation as a general shift in the entire population of $\mathrm{CD} 45^{-}$and $\mathrm{CD} 45^{+}$cells (Figure $3 \mathrm{C}$ ), and the intensity of PD-L1 staining was the same between virus-infected and noninfected cells (Figure $3 \mathrm{H}$ ), suggesting that the effect was not mediated by a direct infection by the virus.

Given these findings and the short interval from infection to the general PD-L1 upregulation in the infected tumors, we reasoned that the effect was probably mediated by locally secreted innate immune mediators, produced in response to direct infection of tumor cells or tumor-infiltrating immune cells (Supplemental Figures 1 and 2). In support of this hypothesis, infection of mouse and human tumor cell lines with NDV-GFP in vitro resulted in a marked upregulation of PD-L1 on the surface of both virus-infected and noninfected cells (Figure 4A and Supplemental Figure 5). Furthermore, cell culture supernatants from the infected cells treated with UV light to inactivate the virus induced strong PD-L1 upregulation when transferred to noninfected cells (Figure 4B), suggesting that the observed upregulation of PD-L1 was primarily due to secretion of immune factors acting on other cells in a paracrine fashion.

Given our observations in cell culture in the absence of an adaptive immune response, we hypothesized that type I IFN played a major role in the early PD-L1 upregulation that occurred in response to NDV. Infection of B16-F10 cells resulted in high expression of type I IFN transcripts after 24 hours (Figure 4C). We also detected secretion of IFN- $\beta$ and TNF- $\alpha$ after 24 hours of infection of human tumor histocultures (Figure 4D), although we were unable to detect additional cytokines, such as GM-CSF, IL-1b, IL-2, IL-6, IL-8, IL-10, or IL-12. Treatment of B16-F10 cells with type I IFN resulted in efficient surface upregulation of PD-L1 after 24 hours (Figure 4E). To determine whether type I IFN was the sole regulator of the PD-L1 increase, we treated cell culture supernatants from NDV-infected cells with UV light to inactivate any virus and then transferred the NDV-free supernatant to B16-F10 cells preincubated with an antibody blocking the IFNAR. Anti-IFNAR antibody treatment resulted in a complete abrogation of PD-L1 upregulation by NDV (Figure 4F), confirming that type I IFN is responsible for the NDV-mediated PD-L1 upregulation in vitro. These findings do not exclude the contribution of other cytokines upregulated by NDV in vivo, especially in view of the additional innate immune response genes that we found to be upregulated in human tumor explants shortly after NDV infection.

We next sought to determine whether type I IFN could be sufficient for PD-L1 upregulation and a therapeutic effect mediated by NDV in vivo. Mice bearing bilateral B16-F10 melanoma tumors were treated intratumorally with recombinant IFN- $\alpha$ or NDV in a single flank (Supplemental Figure 6A). Treatment with IFN- $\alpha$ resulted in the upregulation of PD-L1 on both tumor cells and tumor-infiltrating leukocytes in the treated tumors (Supplemental Figure 6B), thus suggesting a potential role for a type I IFN response in early upregulation of PD-L1 in a direct response to viral infection. In contrast, our analysis of distant tumors revealed no PD-L1 upregulation with IFN- $\alpha 1$ treatment (Supplemental Fig- ure 6C). Furthermore, while both IFN- $\alpha$ and NDV treatment controlled the growth of the injected tumor, only NDV treatment led to a delayed outgrowth of distant tumors (Supplemental Figure 6). To examine the effect of intratumoral type I IFN on tumorspecific lymphocyte infiltration, animals bearing bilateral flank B16-F10 tumors were treated with NDV or IFN- $\alpha 1$ in the rightflank tumor as above and received adoptively transferred transgenic firefly luciferase-expressing $\mathrm{CD}^{+}$lymphocytes recognizing the melanoma Trp1 antigen (herein referred to as Trp1 lymphocytes) (Supplemental Figure 6A). Intratumoral treatment with NDV resulted in increased Trp1 lymphocyte infiltration in both virus-injected and distant tumors, an effect that was not seen with IFN- $\alpha$ (Figure $5, \mathrm{~A}-\mathrm{C}$ ). Finally, we observed an increase in $\mathrm{T}$ cell infiltration into distant tumors with NDV treatment but not with intratumoral IFN- $\alpha$ therapy (Figure 5D).

These findings highlight the idea that, while NDV-induced early cytokine responses could drive local early PD-L1 expression, delayed PD-L1 upregulation in distant tumors is probably driven by a different mechanism and possibly represents an adaptive immune resistance mechanism in response to increased intratumoral immune cells (Figure 5, A-D), as was demonstrated previously (22-24). Indeed, CD274 gene expression strongly correlated with the expression of $C D 8 a$ in the treated distant tumors (Figure $5 \mathrm{E}$ ), and the samples with the highest levels of $\mathrm{CD}^{+} \mathrm{T}$ cell infiltration by flow cytometry showed the highest levels of PD-L1 protein on both $\mathrm{CD}^{-} 5^{-}$and myeloid cells (Figure $5 \mathrm{~F}$ ). To analyze the upregulation of PD-L1 and its temporal-spatial association with immune infiltrates, we collected distant tumors from the NDV-treated animals on days 1 through 5 after a single NDV treatment and processed them for immunohistochemical analysis. We observed a progressive increase in the density of $\mathrm{T}$ cells within the tumor starting 3 days after treatment (Figure $5 \mathrm{G}$ ), with an associated increase in $\mathrm{PD}-\mathrm{L}^{+}$staining (Figure $5 \mathrm{H}$ ). Consistent with the flow cytometric findings, we observed PD-L1 staining primarily in the tumor-infiltrating myeloid cells, with a minor increase in the intensity of PD-L1 staining on the tumor cells (Figure 5H).

Combination therapy with intratumoral NDV and systemic PD-1 or PD-L1 blockade results in rejection of the treated and distant tumors. The findings described above suggest that with NDV treatment, upregulation of PD-L1 in the tumor microenvironment happens early in the virus-injected tumors as a result of an innate immune response to viral infection, and later in distant tumors as a response to immune infiltration. To evaluate whether the PD-1/ PD-L1 pathway acts as a resistance mechanism preventing complete tumor rejection by NDV therapy, we proceeded to examine the efficacy of intratumoral NDV therapy in combination with systemic PD-1/PD-L1 blockade. Since PD-L1 upregulation was detected early after NDV infection, treatment with anti-PD-1 and anti-PD-L1 antibodies was initiated concomitantly with NDV treatment. Animals bearing bilateral B16-F10 tumors were treated with NDV, which was injected into a single flank tumor and received concomitant systemic PD-1- or PD-L1-blocking antibody. The combination approach resulted in complete regression of both NDV-injected and distant tumors in the majority of the treated animals, an effect that was not seen with either treatment alone (Figure 6, A and B). This led to long-term survival in the majority of the animals treated with combination therapies (Figure $6 \mathrm{C}$ ). We 
A

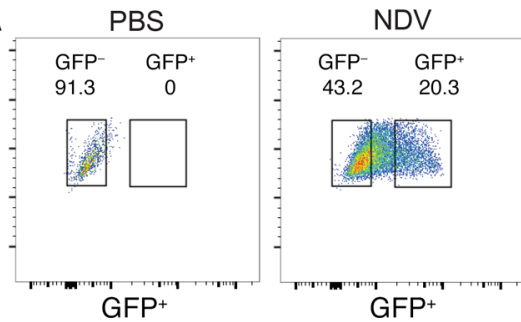

B

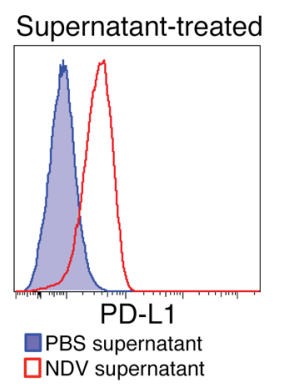

E

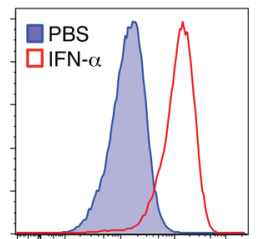

PD-L1
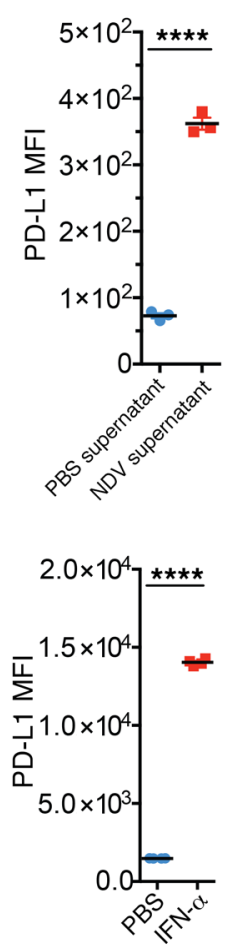

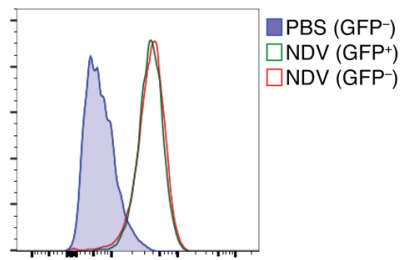

PD-L1
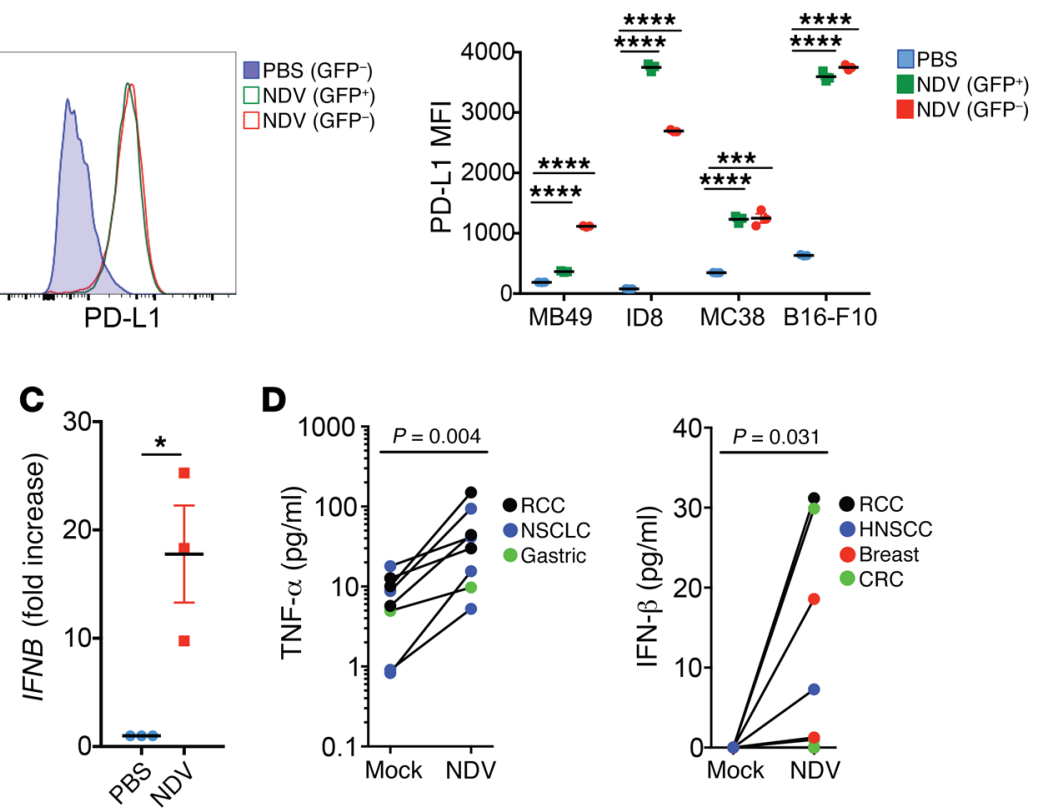

$\mathbf{F}$
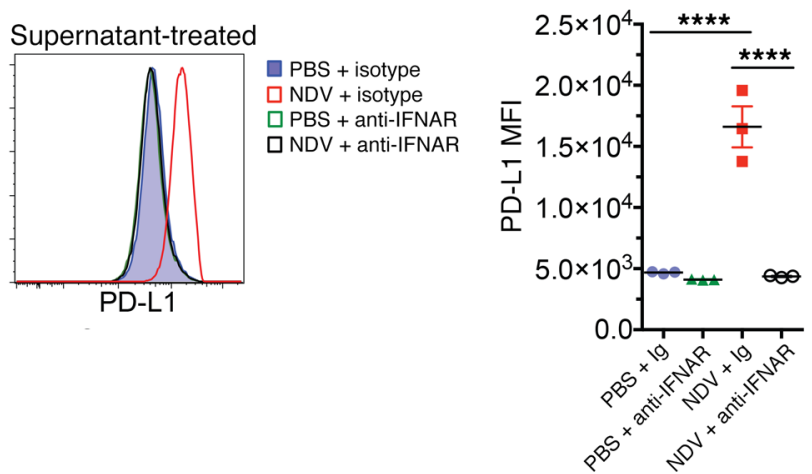

Figure 4. Upregulation of PD-L1 in tumor cells by NDV-induced type I IFN. (A) PD-L1 expression in the infected (GFP') and noninfected (GFP-) B16-F10 cells treated with NDV-GFP in vitro. Shown are representative flow cytometric plots from B16-F10 cells and quantification of PD-L1 MFI from different infected cell lines. (B) PD-L1 upregulation in B16-F10 cells treated with UV-inactivated supernatant from NDV-infected cells. (C) Expression of IFNB in NDV-infected B16-F10 cells determined at 24 hours by RT-PCR. (D) Production of innate cytokines in human tumor histoculture in response to NDV determined by ELISA at 24 hours. NSCLC, non-small-cell lung cancer. (E) Upregulation of PD-L1 in B16-F10 cells in response to treatment with recombinant IFN- $\alpha 2$. (F) Inhibition of PD-L1 upregulation by anti-IFNAR antibody in B16-F10 cells treated with UV-inactivated supernatant from NDV-infected cells. (A-C and F) Results are representative of 3 independent experiments with 3 replicates per group. Data indicate the mean \pm SEM. (D) Each tumor specimen represents an individual experiment. Data were analyzed by Student's $t$ test for individual comparisons (A-C) and Wilcoxon matched-pairs, signed-rank test (D). ${ }^{*} P<0.05,{ }^{* * *} P<0.01$, and ${ }^{* * * *} P<0.0001$.

observed a similar effect in a bilateral-flank CT26 colon carcinoma mouse model (Supplemental Figure 7, A-C). Finally, we evaluated the efficacy of combination therapy in a poorly immunogenic genetically engineered $B R A F^{V 600 E} P^{6 T E N}{ }^{\operatorname{loxP} / 10 \times P}$-inducible melanoma model (25). Animals with induced established bilateral-flank tumors were treated with intratumoral NDV in a single tumor in combination with systemic PD-1 blockade. The combination of NDV with PD-1 blockade resulted in a significant delay in the growth of both virus-injected and distant tumors compared with PD-1 blockade alone (Supplemental Figure 7, D and E).

To determine whether the combination of PD-1 blockade with NDV could lead to long-term memory formation, animals that completely cleared B16-F10 tumors and survived more than
60 days were reimplanted with $2 \times 10^{5}$ B16-F10 cells without further treatment. We found that a significant delay in tumor growth occurred in the majority of animals and that a subset of animals was completely protected from the tumor reimplantation (Figure 6D), although the degree of protection was lower than that seen in our previous studies using combined NDV treatment and CTLA4 blockade (5). While these findings could be explained by the experimental differences, they may also suggest that the expansion of $\mathrm{T}$ cells with an effector phenotype within the context of PD-1 blockade may have a different impact on the formation of long-term tumor-specific memory $(26,27)$.

Combination therapy with intratumoral NDV and systemic PD-1 blockade promotes distant tumor infiltration with activated $T$ cells. 
A
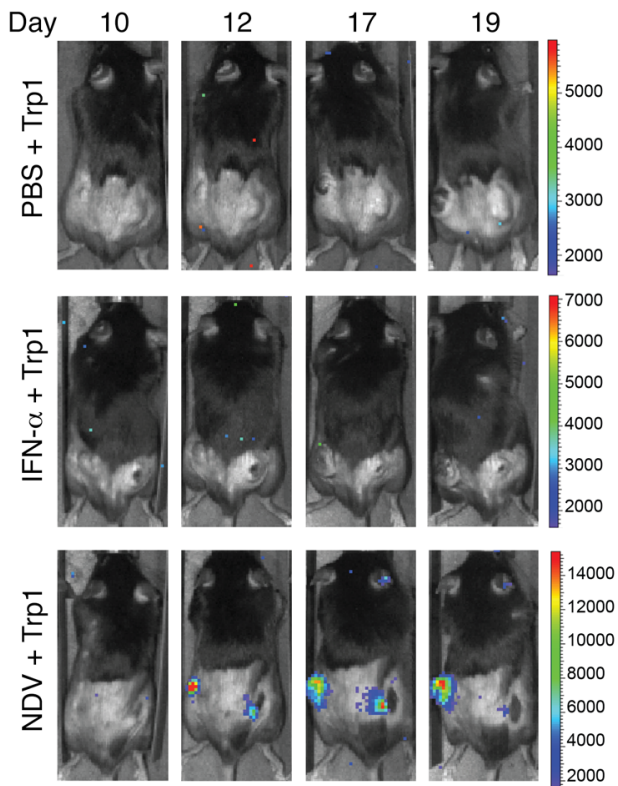

B
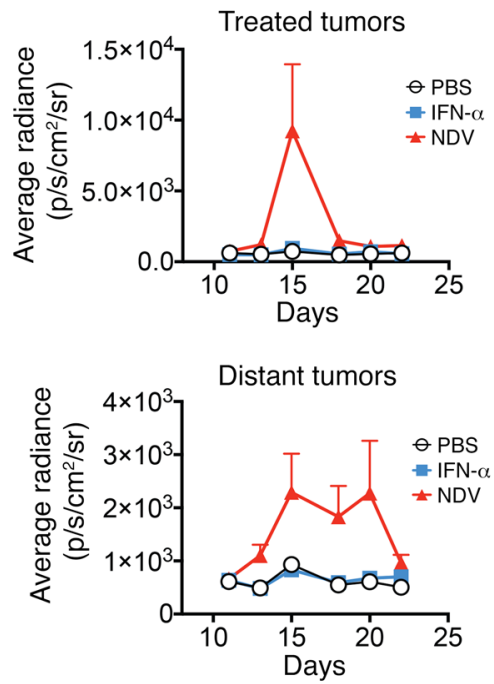

C
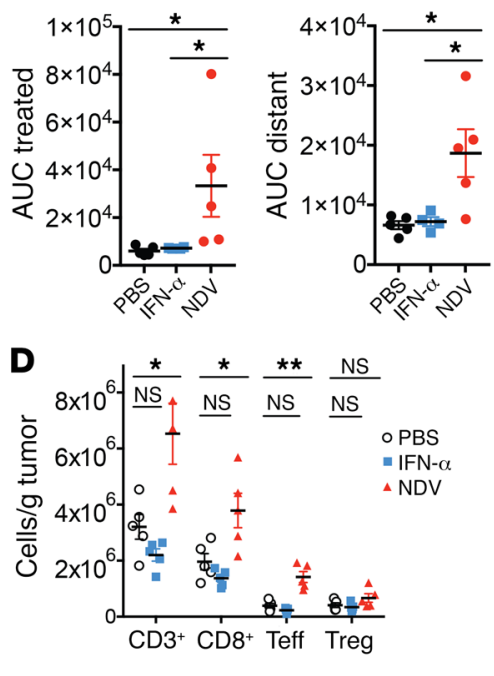

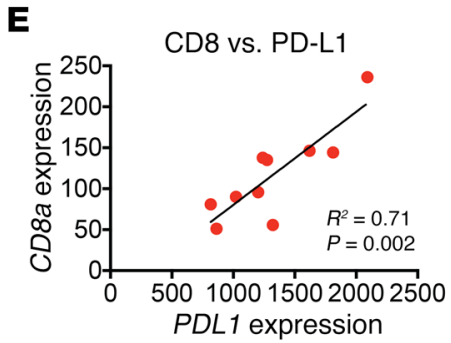

G

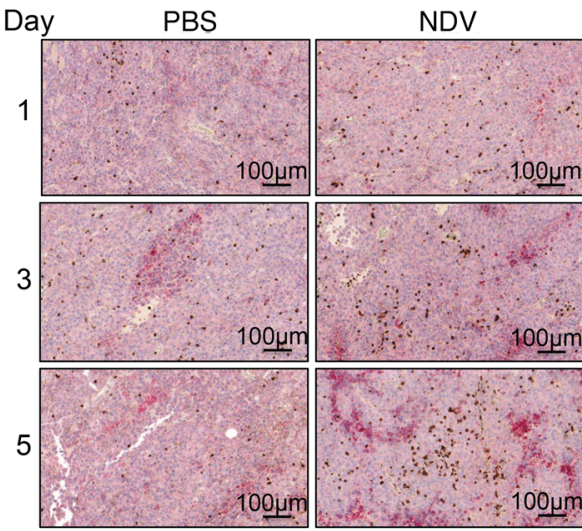

Red: PD-L1 Brown: CD3
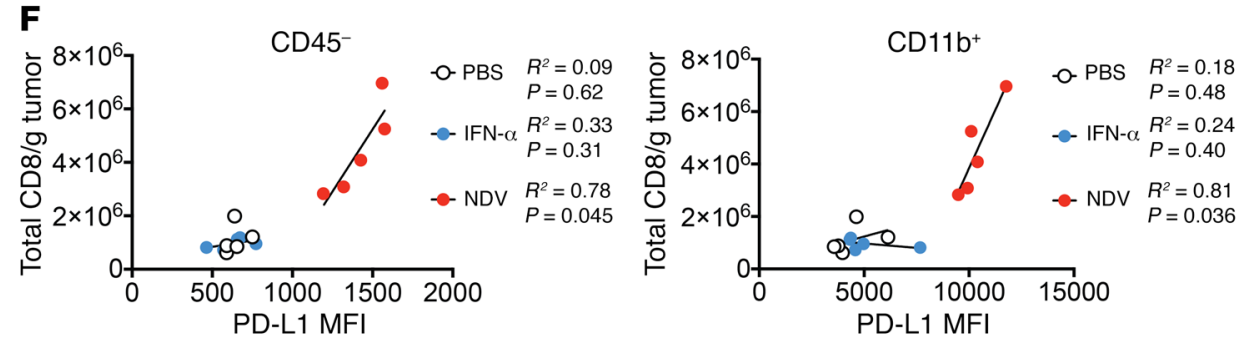

H
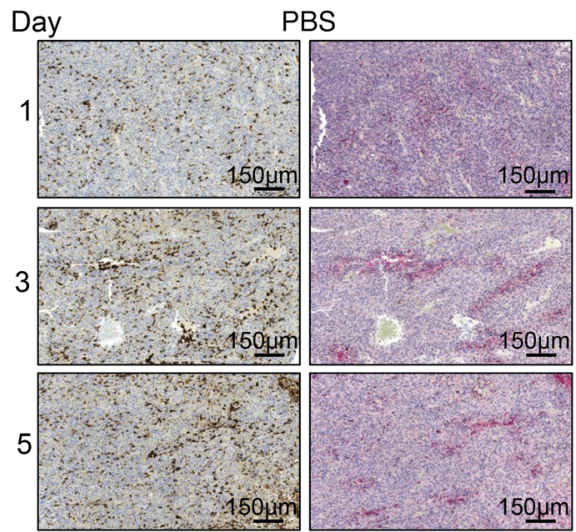

CD11b

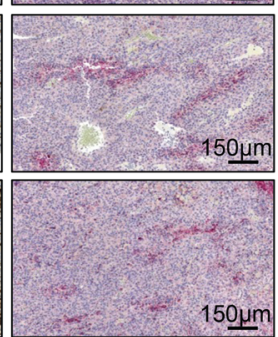

PD-L1
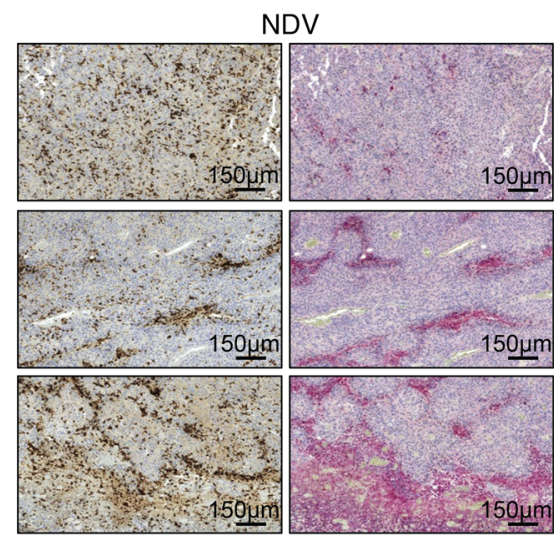

CD11b

PD-L1

Figure 5. Role of innate and adaptive immune responses in NDV-induced PD-L1 upregulation. Animals were treated as shown in the schema in Supplemental Figure 5. (A) Infiltration of tumors with adoptively transferred Trp1-luc lymphocytes with intratumoral NDV or IFN- $\alpha$ therapy. (B) Quantification of the average radiance from $\mathbf{A}$ in treated and distant tumors. (C) Quantification of the AUC of luminescence in treated and distant tumors. (D) Immune infiltration in distant tumors with NDV versus IFN- $\alpha$ treatment calculated using flow cytometry. Teff, effector T cell. (E) Association of $P D L 1$ gene expression with CD8a gene expression in distant tumors from NDV-treated animals. (F) Association of PD-L1 expression on CD45 cells and CD11 $\mathrm{b}^{+}$cells with total CD8 ${ }^{+}$ infiltration in distant tumors calculated using flow cytometry. (G) Time course of T cell infiltration and PD-L1 upregulation in distant tumors in response to single NDV injection. Scale bar: $100 \mu \mathrm{m}$. (H) Association of PD-L1 upregulation with myeloid cell infiltration into distant tumors over time. Scale bar: 150 $\mu \mathrm{m}$. Results are representative of 2 to 3 independent experiments with 5 to 10 mice per group, and data represent the mean \pm SEM. Data were analyzed by Student's $t$ test for individual comparisons (C and $\mathbf{D}$ ) and Pearson's correlation (E and $\mathbf{F}){ }^{*} P<0.05,{ }^{*} P<0.01$. 
A
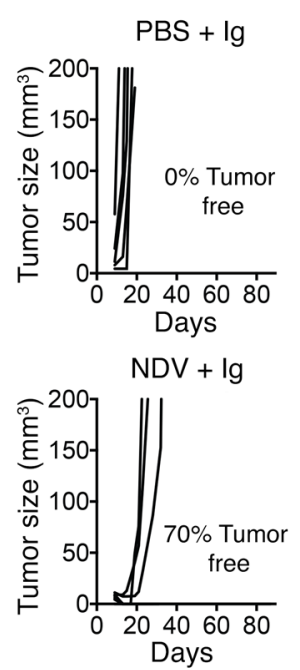

Treated tumors

PBS + anti-PD-1
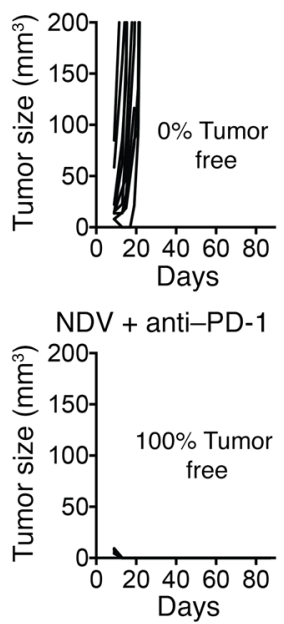

PBS + anti-PD-L1
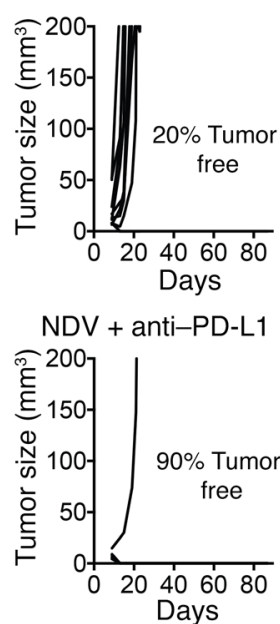

B
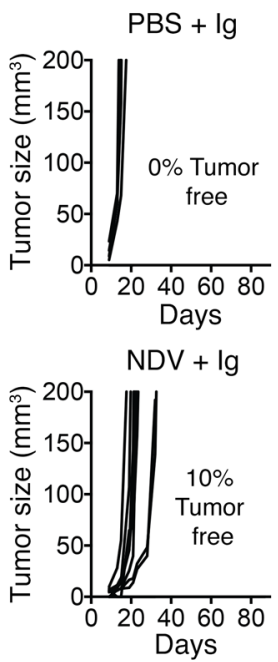

Distant tumors
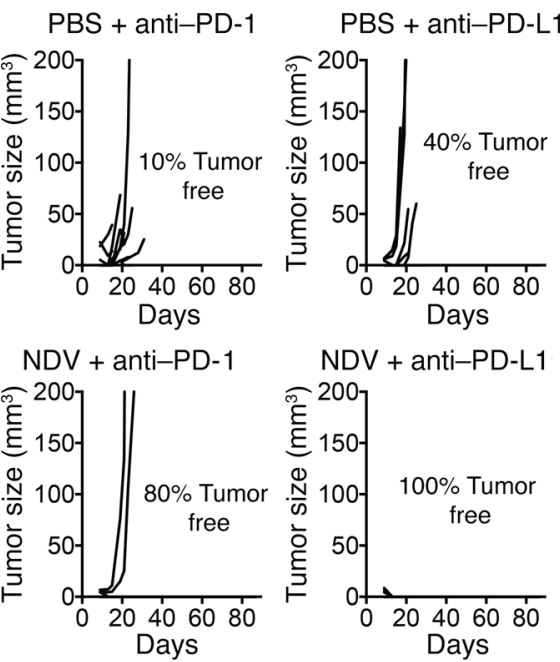

C

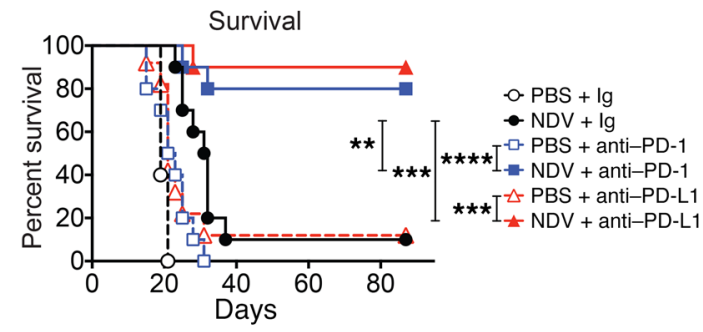

D

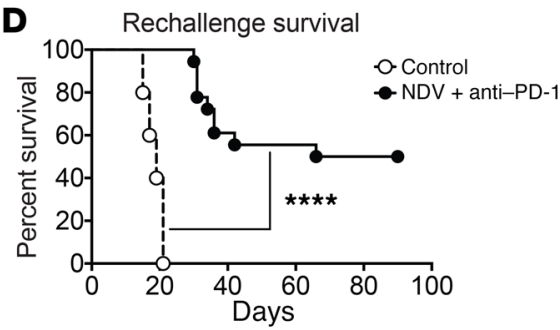

Figure 6. Local and abscopal effects of intratumoral NDV in combination with systemic PD-1 or PD-L1 blockade. B16-F10 tumors were implanted by i.d. injection of $4 \times 10^{5}$ B16-F10 cells into the right flank and $5 \times 10^{4}$ cells into the left flank on day 0 . On days 3, 5, 7, and 9, mice were treated with intratumoral injections of NDV or PBS and concomitant i.p. injections of anti-PD-1, anti-PD-L1, or isotype control antibody. (A) Growth of NDV-treated tumors. (B) Growth of distant tumors. (C) Overall survival. (D) Rechallenge of surviving animals at 90 days. (A and B) Data represent 1 of 2 experiments with 10 mice per group. (C and $\mathbf{D})$ Data represent 2 pooled experiments with 10 mice per group. ${ }^{* *} P<0.01,{ }^{* * *} P<0.001$, and ${ }^{* * *} P<0.0001$, by log-rank test $(\mathbf{C}$ and $\mathbf{D})$.

Gene expression analysis from bilateral tumors revealed the upregulation of genes related to T cell infiltration in both NDV-treated and distant tumors, with the most pronounced effect observed in the combination treatment group (Figure 7, A and B). We detected an increase in the transcriptional upregulation of genes related to $\mathrm{T}$ cell activation, including the $\mathrm{T}$ cell costimulatory receptors TNFA and IFNG (Figure 7, A and B). The increases were observed in both virus-injected and distant tumors, but not in the spleen (Figure 7, $A$ and B, and Supplemental Figure 8), suggesting that the observed inflammatory effects were selective for the tumor and not a reflection of a nonspecific systemic inflammatory response. To characterize the effects of therapy on the tumor microenvironment, cells from distant tumors of the animals treated with intratumoral NDV, systemic anti-PD-1, or a combination of both were isolated and analyzed by flow cytometry. Combination therapy resulted in a significant increase in the number of infiltrating $\mathrm{CD} 8^{+}$and Tcon cells (Figure 7, C and D), with a relative decrease in the percentage of intratumoral Tregs (Figure 7E), resulting in enhanced effector/Treg ratios (Figure 7F). Analysis of the infiltrating T cells showed an upregulation of Ki-67, GrB, and ICOS, an effect that was most pronounced in the animals treated with combination therapy (Figure 7G and Supplemental Figure 9). To look for evidence of tumor-specific immunity, tumor-infiltrating $\mathrm{CD}^{+}$cells isolated from distant tumors were restimulated with DCs pulsed with B16-F10 tumor lysates and assessed by intracellular cytokine staining. The strongest increase in IFN- $\gamma$ production in response to antigen stimulation occurred with combined NDV and PD-1 blockade treatment (Figure 7H). NDV therapy led to the downregulation of PD-1 expression on tumor-infiltrating Tregs, Tcon cells, and $\mathrm{CD}^{+}$cells, which was most pronounced in the combination therapy group (Supplemental Figure 10A). Notably, the majority of the $\mathrm{GrB}^{+} \mathrm{CD}^{+}$lymphocytes were in the $\mathrm{PD}-1^{-}$fraction, which was a reversal from the untreated animals and the those treated with a single-agent anti-PD-1 antibody (Supplemental Figure 10B). We observed a similar expansion in the $\mathrm{CD}^{+}{ }^{+} \mathrm{FoxP}^{-}$(Tcon) cell subset (Supplemental Figure 10B). Further studies will be needed to carefully examine the function of the PD- $1^{+}$and $\mathrm{PD}-1^{-} \mathrm{T}$ cell subsets generated in response to such therapy. Overall, these findings suggest that the combination of NDV and PD-1 blockade leads to an expansion of activated effector $\mathrm{T}$ cell populations that are most likely responsible for the increase in therapeutic efficacy.

Efficacy of NDV therapy with PD-1 blockade is dependent on $C D 8^{+}$cells and NK cells. To determine the cellular subsets that are necessary for the observed therapeutic effect with combination therapy, animals bearing bilateral B16-F10 tumors received combination therapy with NDV and PD-1 blockade starting on day 3 and, in addition, were treated with depleting antibodies against CD8, CD4, and NK cells. To determine the role of the particular 
A

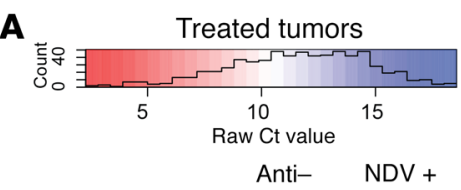

$\begin{array}{lll}\text { PBS NDV } & \text { Anti- } & \text { PD-1 } \\ \text { anti-PD-1 }\end{array}$

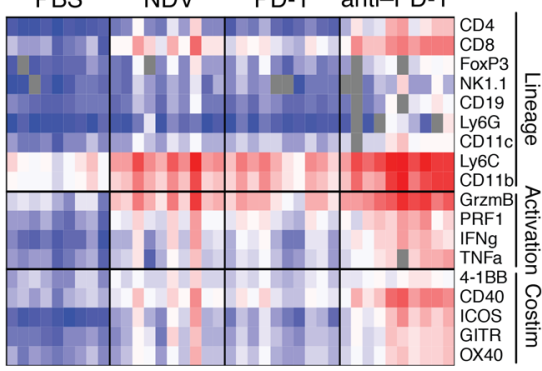

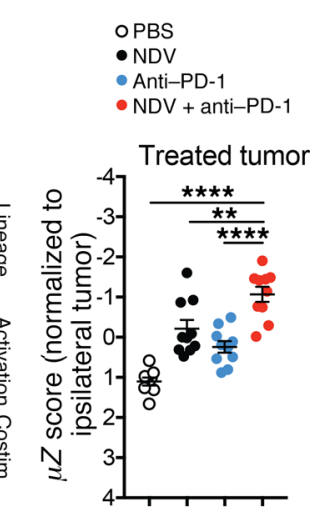

$\mathrm{PBS}+$

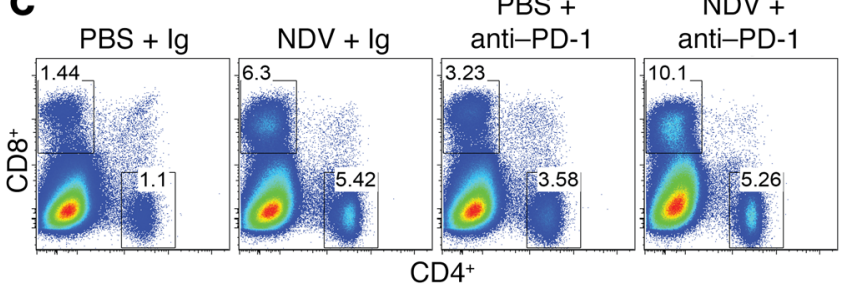

D

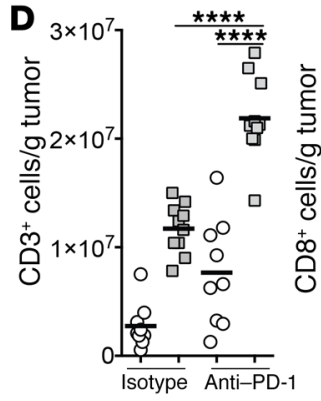

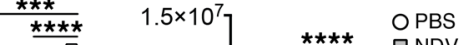

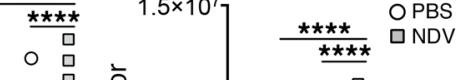

$\mathbf{F}$

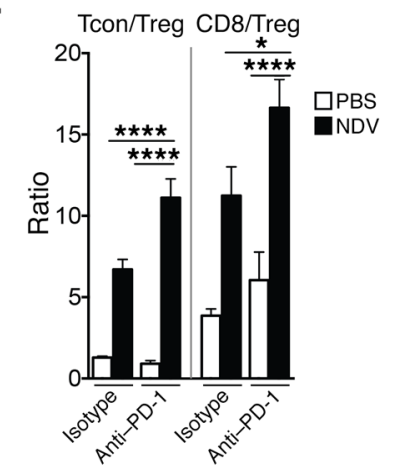

B Distant tumors
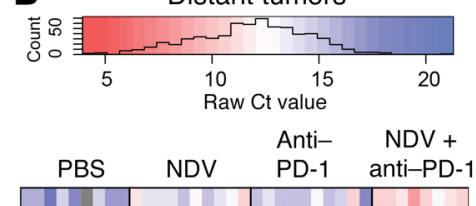

OPBS

- NDV

- Anti-PD-1

- NDV + anti-PD-1
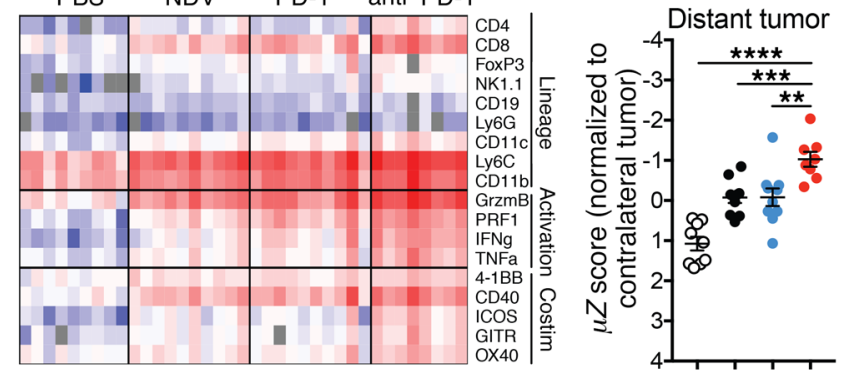

$\mathrm{PBS}+$

$\mathrm{NDV}+$

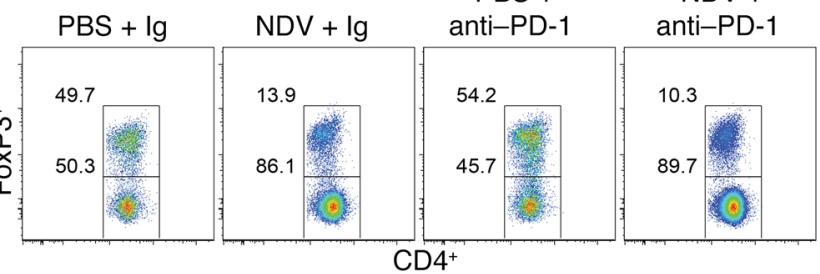

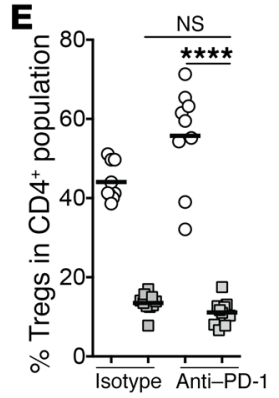

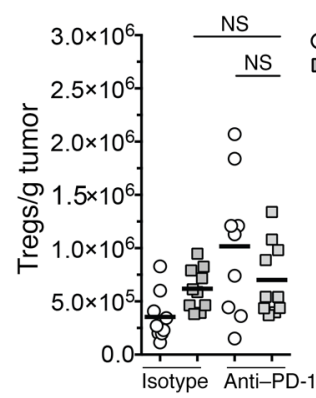

G
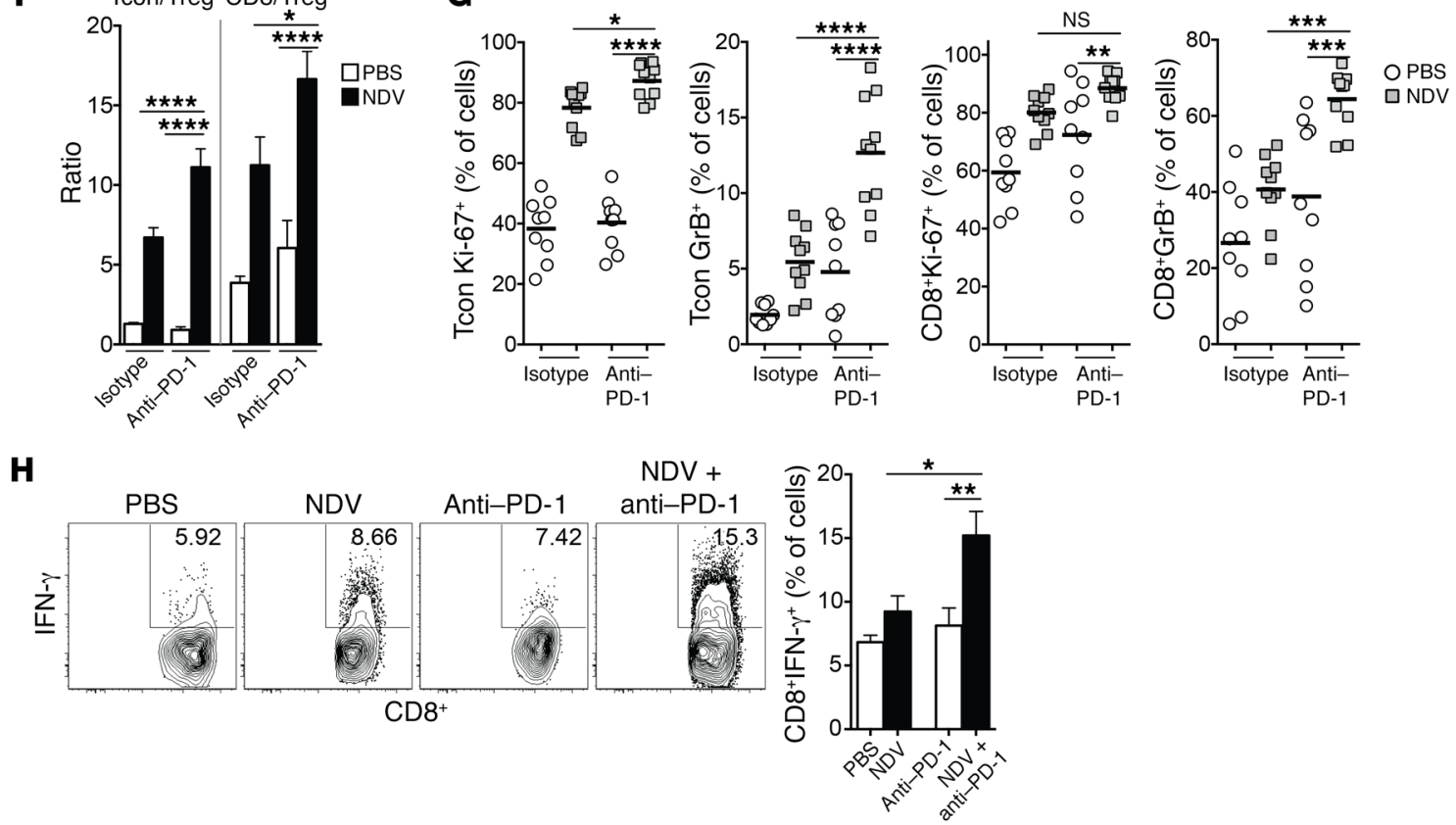
Figure 7. Potentiation of immune effects of NDV by PD-1 blockade. Animals bearing bilateral flank B16-F10 melanoma tumors were treated according to the schedule in Figure 6. (A and $\mathbf{B}$ ) Gene expression analyses from treated $(\mathbf{A})$ and distant $(\mathbf{B})$ tumors, focusing on selected lineagedefining and $\mathrm{T}$ cell activation and costimulation markers. Costimulation (Costim) and activation markers were used to calculate an activation signature $Z$ score. (C) Representative plots of percentages of $C D 4^{+}$and CD8 ${ }^{+}$Iymphocytes from distant tumors (gated on total live cells). (D) Absolute numbers of $\mathrm{CD}^{+}, \mathrm{CD}^{+}$, and $\mathrm{CD}^{+} \mathrm{FoxP3}^{-}$(Tcon) lymphocytes in distant tumors. (E) Relative percentages and absolute numbers of Tregs in distant tumors. (F) Tcon/Treg and CD8 $8^{+} /$Treg ratios in distant tumors. (C) Expression of proliferation and lytic markers by tumor-infiltrating CD8 and Tcon cells in distant tumors. (H) IFN- $\gamma$ release by distant tumor-infiltrating CD8 $8^{+}$Iymphocytes in response to stimulation with tumor antigen-loaded DCs. Data represent 1 of 2 experiments with 10 mice per group and indicate the mean \pm SEM. Data were analyzed by 1-way ANOVA with multiple comparisons. ${ }^{*} P<0.05,{ }^{* *} P<0.01,{ }^{* *} P<0.001$, and ${ }^{* * *} P<0.0001$.

cell subtypes early versus late in the treatment course, the depleting antibodies were injected starting either before implantation (day -1) or concomitantly with the first treatment (day 3). Depletion before tumor implantation showed that both $\mathrm{NK}$ and $\mathrm{CD} 8^{+}$ cells were essential for a therapeutic effect (Figure 8A), although this experiment did not distinguish the potential role of NKT cells, which may play an effector or regulatory role. When the depletion was started on the first day of treatment, only $\mathrm{CD} 8^{+}$cells proved to be essential for efficacy (Figure 8B). These results suggest that NK cells are important very early on in the treatment course, possibly as immediate responders to NDV treatment. NDV has been previously demonstrated to activate NK cells (28), and we hypothesize that NK cells may be responsible for early inflammatory responses and IFN- $\gamma$ production, while $\mathrm{CD}^{+}$cells are responsible for longterm antigen-specific tumor control. As treatment with anti-NK1.1 antibody probably does not result in an immediate depletion of NK cells, it is likely that early activation of the NK cells in response to NDV is sufficient to drive a further therapeutic response when NK cells are depleted at a late stage of treatment. Interestingly, we did not observe a significant detriment to therapeutic efficacy with $\mathrm{CD} 4$ depletion in either setting, though this finding must be interpreted with caution, as both conventional and regulatory $\mathrm{T}$ cell subsets were depleted in this setting.

\section{Discussion}

OVs represent a promising class of emerging cancer therapeutics, with recent studies focusing on the immunotherapeutic rather than the strictly lytic properties of these agents. The presumed mechanism of action of OV immunotherapy can be attributed to immunogenic cell death, with upregulation of MHCs and the release of pathogen-associated molecular pattern molecules (PAMPs), dangerassociated molecular patterns (DAMPs), and other inflammatory mediators driving activation not just of antiviral but also of antitumor immuni- ty (29-32), which can lead to local and abscopal effects. While serving as an attractive in situ vaccination strategy, intratumoral oncolytic virotherapy by itself appears to be of limited therapeutic value.

NDV is an avian paramyxovirus that exhibits a broad tropism for different cell and cancer types because of the ubiquitous expression of sialic acid, which serves as a receptor for the virus $(4,33-37)$. Previous studies involving i.v. administration of NDV demonstrated durable responses in some patients that were maintained after treatment discontinuation, which is suggestive of an immune-mediated therapeutic effect (38). Several studies have shown that the compensatory immune-inhibitory mechanisms induced in the tumor microenvironment by the immune response can dampen the efficacy of immunotherapy and that effective blockade of such mechanisms through combination therapies can result in a better therapeutic effect $(22,39)$. In the current study, we set out to identify the potential mechanisms of resistance to NDV-induced immune activation. We found that NDV activated a range of immune-inhibitory pathways including PD-L1 in both infected tumors and in tumors not infected by the virus. Our findings suggest that, within the context of NDV therapy, PD-L1 upregulation occurs early in the virus-injected tumors as a paracrine response to the innate immune stimuli induced by the virus, and later as an adaptive immune resistance mechanism against immune infiltration.

Multiple trials using PD-1- and PD-L1-blocking antibodies in different cancer types have demonstrated that tumor positivity for PD-L1 is enriched in patients who are more likely to respond to PD-1 and PD-L1 blockade $(23,40,41)$. As a result, it has been previously suggested that for PD-L1- tumors, strategies that could induce PD-L1 upregulation would enhance the efficacy of immune checkpoint blockade (42). In support of this, our findings show that intratumoral therapy with NDV sensitized the tumors to the efficacy of PD-1 and PD-L1 blockade in tumor models that were otherwise not responsive to such a strategy. Notably, in human tumor explants, NDV therapy upregulated multiple immunerelated genes that have recently been shown to predict a response to anti-PD-1 antibodies in clinical trials (14).

Several OVs are currently being explored in a similar fashion in the clinical setting, with early data showing highly promising results with CAVATAK and T-vec $(8,43)$. Several studies have in addition recently examined combinations of OVs with PD-1 or

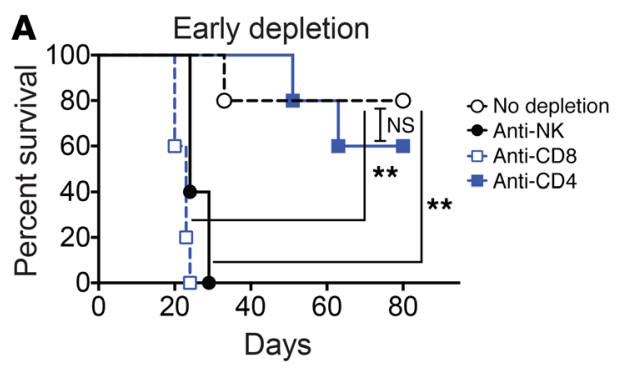

Figure 8. Dependence of NDV and PD-1 blockade on NK cells and CD8+ Iymphocytes. Bilateral B16-F10 tumors were established, and animals were treated with NDV and anti-PD-1 antibody as described in Figure 6 in the presence of the indicated depleting antibodies. (A) Survival of animals that received the indicated depleting antibodies before tumor implantation. (B) Survival of animals that received the indicated depleting antibodies on day 0 of therapy. Data are representative of 2 experiments with 5 mice per group.

${ }^{* *} P<0.01$, by log-rank test.

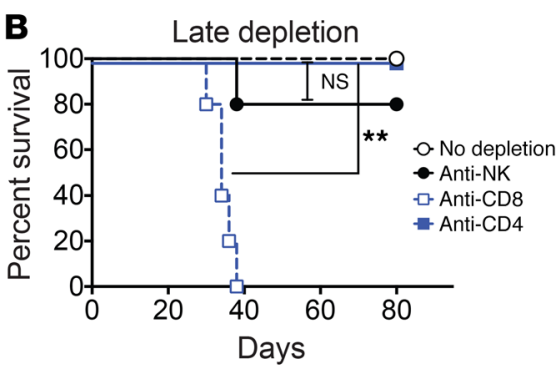

jci.org Volume $128 \quad$ Number 4 April 2018

1423 
PD-L1 blockade in animal models, although most of the studies primarily focused on analyses of virus-injected tumors, making it difficult to dissect the antiviral versus antitumor immune responses (44-49). Interestingly, results among the different studies are somewhat divergent. While therapy with vesicular stomatitis virus (VSV) in combination with adoptive cell therapy resulted in the expansion of $\mathrm{PD}-1^{+} \mathrm{CD} 8^{+} \mathrm{T}$ cells, the addition of anti-PD- 1 therapy did not improve the efficacy of this therapeutic approach (50). Intratumorally administered reovirus in combination with systemic PD-1 blockade resulted in synergistic tumor growth inhibition, which was dependent on $\mathrm{NK}$ and $\mathrm{CD}^{+}$cells (47). Interestingly, in contrast to our findings with NDV, no significant PD-L1 induction was seen in that study in response to reovirus alone, and the induction of PD-L1 was attributed to the action of tumor-infiltrating NK cells (47). A study of combined oncolytic vaccinia and PD-L1 blockade demonstrated synergistic efficacy against the virus-treated peritoneal tumors, and the efficacy of such therapy was dependent on both $\mathrm{CD}^{+}$and $\mathrm{CD} 8^{+}$cells (44). Recombinant HSV expressing IL-12 combined with PD-1 blockade in a glioma model revealed a dependency on $\mathrm{CD}^{+}$and $\mathrm{CD} 8^{+} \mathrm{T}$ cells as well as macrophages (49). The discrepancies between these studies can probably be attributed to the biological differences between the different viruses and highlight the notion that results with $1 \mathrm{OV}$ cannot necessarily be extrapolated to all OVs as a therapeutic class.

The present study, among others, demonstrates that inflammatory responses and PD-L1 upregulation in tumors with OV therapy can sensitize these tumors to PD-1/PD-L1 blockade. It therefore raises the question of whether monitoring patients in vivo for evidence of local and abscopal inflammatory responses could aid clinical decision making during OV therapy (e.g., therapeutic sequencing and determination of the number of intratumoral injections needed). In our study, imaging of animals treated intratumorally with NDV and with adoptively transferred luciferase-expressing $\mathrm{T}$ cells revealed increased $T$ cell infiltration $4-5$ days after NDV treatment (Figure 5). In a trial conducted by Streby et al., intratumoral administration of HSV1716, an oncolytic herpes virus, showed transient increases in fluorodeoxyglucose-PET (FDG-PET) activity in some patients, consistent with a tumor inflammatory reaction (51). This creates a strong rationale for in vivo monitoring of tumor infiltration, perhaps specifically by imaging $\mathrm{CD} 8^{+}$lymphocytes. Indeed, a recent study in animal models has demonstrated that in vivo monitoring of tumor CD8 infiltration using a ${ }^{89} \mathrm{Zr}$-labeled anti-CD8 antibody fragment could predict therapeutic efficacy in the context of anti-CTLA-4 therapy (52).

In conclusion, our findings suggest that PD-L1 plays a key role in resistance to NDV-induced antitumor immune responses on both local and systemic levels. These findings have implications for the timing of PD-1/PD-L1 blockade in conjunction with OV therapy and highlight the importance of understanding the adaptive mechanisms of immune resistance to specific OVs for the rational design of appropriate combinatorial approaches with these agents.

\section{Methods}

Mice. C57BL/6J mice were purchased from The Jackson Laboratory. The Braf-transgenic mice (TyrCre-Braf ${ }^{V 600 E}$ Pten $^{K O}$ ) were initially developed by the McMahon and Bosenberg laboratory (25) and were backcrossed onto a C57BL/6 background. Topical application of hydroxytamoxifen gave rise to melanoma tumors starting 30 days after induction. All mice were maintained in microisolator cages.

Human tumor histoculture. Fresh tumor tissues were collected within 1 hour of surgery and transported overnight at $4^{\circ} \mathrm{C}$ to MRL. The tumors were embedded in $1 \%$ low-melting gel and cut into $400-\mu \mathrm{m}$ slices using the McIlwain Tissue Chopper. The tumor slices were set onto Millicell-CM inserts in 6-well dishes containing $1 \mathrm{ml} \mathrm{DMEM}$, $10 \% \mathrm{FBS}, 100 \mathrm{U} / \mathrm{ml}$ penicillin, and $100 \mu \mathrm{g} / \mathrm{ml}$ streptomycin. Renal cell carcinoma (RCC), colorectal cancer (CRC), breast cancer, head and neck squamous cell carcinoma (HNSCC), and gastric carcinoma tumor specimens were treated with NDV $\left(3 \times 10^{7} \mathrm{PFU}\right)$. After 24 to 48 hours of incubation, tissue slices were collected for RNA extraction and gene expression analysis. Supernatants were collected at 24 hours for IFN- $\beta$ detection using a Human IFN- $\beta$ Tissue Culture Kit (MSD).

Ex vivo treatment of whole blood with NDV. Whole blood from patients with cancer and from healthy donors was collected into K2-ETDA-coated tubes and held at $4^{\circ} \mathrm{C}$ until use. The blood was then incubated with NDV $\left(3 \times 10^{7} \mathrm{PFU} / \mathrm{ml}\right.$ blood $)$ in standard tissue culturetreated plates in a $37^{\circ} \mathrm{C}, 5 \% \mathrm{CO}_{2}$ incubator. After 24 hours, a portion of the blood was centrifuged at 1,000 to $1,500 \mathrm{~g}$ for 15 minutes to separate out the plasma. The plasma was removed and snap-frozen for future protein analysis. The remainder of the blood was collected into PAXgene blood RNA tubes, inverted until well mixed, and allowed to sit at room temperature for 2 hours before freezing at $-20^{\circ} \mathrm{C}$. The plasma was assessed for IFN- $\beta$ using MSD technology. RNA from blood was isolated according to the manufacturer's protocol and the gene expression assessed as outlined below.

Cell lines. The murine cancer cell lines for melanoma (B16-F10, originally provided by Isaiah Fidler, MD Anderson Cancer Center, Houston, Texas, USA), colon cancer (CT26 and MC38, originally obtained from ATCC), ovarian cancer (ID8, originally generated by Katherine Roby, University of Kansas Medical Center, Kansas City, Kansas, USA), and bladder carcinoma (MB49, originally generated by Ian Summerhayes, Imperial Cancer Research Fund, London, United Kingdom) were maintained in RPMI medium supplemented with 7.5\% FCS and penicillin with streptomycin. Human melanoma cell lines, originally isolated from patients at the MSKCC (SK-MEL-28, SK-MEL-30, and SK-MEL-31) were maintained in RPMI medium supplemented with $10 \%$ FCS and penicillin with streptomycin. All of the cell lines were tested and found to be negative for mycoplasma contamination. The cell lines were not reauthenticated after their receipt from the original sources.

Antibodies and mouse IFN- $\alpha$. Therapeutic anti-PD-1 (clone RMP114), anti-PD-L1 (clone 9G2), anti-CD8 (clone 2.43), anti-CD4 (clone GK1.5), and anti-NK1.1 (clone PK136) monoclonal antibodies were produced by BioXcell. The following antibodies and dilutions were used for flow cytometric analyses: CD45.2 Alexa Fluor 700 (eBioscience; catalog 56-0454; 1:200); CD3 PE-Cy7 (eBioscience; catalog 25-0031; 1:200); CD4 ef450 (eBioscience; catalog 48-0041; 1:200); CD4 APC-eFluor 780 (eBioscience; catalog 47-0041; 1:400); CD8 PerCP-eFluor710 (eBioscience; catalog 46-0083; 1:200); CD11b APCeFluor 780 (eBioscience; catalog 47-0112; 1:600); ICOS PE (eBioscience; catalog 12-5985; 1:200); PD-L1 PE Cy7 (eBioscience; catalog 25-5982-82; 1:200); NK 1.1 PE (eBioscience; catalog 12-5941; 1:200); IFN- $\gamma$ PE (eBioscience; catalog 12-7311; 1:200); FoxP3 Alexa Fluor 700 (eBioscience; catalog 56-5773; 1:100); FoxP3 APC (eBioscience; 
catalog 17-5773; 1:200); PD-1 PE-Cy7 (eBioscience; catalog 25-9985; 1:200); CD11c APC (eBioscience; catalog 17-0114-82; 1:200); F4/80 eFluor 450 (eBioscience; catalog 48-4801-82; 1:100); Granzyme B PETexas Red (Invitrogen, Thermo Fisher Scientific; catalog GRB17; 1:125); Granzyme B APC (Invitrogen, Thermo Fisher Scientific; catalog GRB05; 1:125); and Ki-67-Alexa Fluor 488 (BD Pharmingen; catalog $561165 ; 1: 50)$. Mouse IFN- $\alpha$ was purchased from Miltenyi Biotec.

Viruses and cloning. The recombinant lentogenic NDV LaSota strain was used for all experiments. To generate NDV virus expressing GFP, a DNA fragment encoding GFP flanked by the appropriate NDVspecific RNA transcriptional signals was inserted into the SacII site created between the P and $\mathrm{M}$ genes of pT7NDV/LS. Viruses were rescued from cDNA using methods described previously and sequenced by reverse transcription PCR for insert fidelity (53). Virus titers were determined by serial dilution and immunofluorescence in A549 cells.

In vitro infection experiments. The cell lines were cultured to $50 \%$ confluency and infected in 6-well dishes at a MOI of 2 in a total volume of $3 \mathrm{ml} \mathrm{RPMI}$ medium supplemented with 10\% FCS and penicillin with streptomycin. Twenty-four hours later, the cells were harvested by scraping and processed for PD-L1 surface labeling and quantification by flow cytometry. Supernatants from the infected and noninfected cells were cleared from debris by centrifugation. The virus present in supernatants was UV inactivated in a Stratalinker 1800 (Stratagene) with 6 pulses of $300 \mathrm{~mJ} / \mathrm{cm}^{2} \mathrm{UV}$ light. The inactivated supernatant was diluted 1:3 in fresh complete RPMI medium and used to treat fresh cells in 6-well plates. Twenty-four hours later, the cells were harvested by scraping and processed for PD-L1 surface labeling and quantification by flow cytometry. For the supernatant transfer experiments involving IFNAR blockade, the supernatants were treated with anti-IFNAR antibody (clone MAR1-5A3; BioXcell) at $10 \mu \mathrm{g} / \mathrm{ml}$ (54). For type I IFN treatment, cells were treated with 2,000 U/ml mouse IFN- $\alpha$ for 24 hours.

Mouse tumor experiments. Bilateral flank tumor models were established to monitor for therapeutic efficacy in both injected and systemic tumors. Bilateral flank B16-F10 melanoma tumors were established by implantation of $2 \times 10^{5}$ cells into the right flank and $5 \times 10^{4}$ cells into the left flank for survival experiments, or $4 \times 10^{5}$ cells into the right flank and $2 \times 10^{5}$ cells into the left flank for TIL and gene expression analyses.

Tumors were established according to the cell dose and schedule indicated in each figure. Intratumoral injections were performed using $2 \times 10^{7} \mathrm{PFU}$ of NDV in PBS in a total volume of $100 \mu \mathrm{l}$. For IFN- $\alpha$ therapy, animals were treated intratumorally with $2 \times 10^{4} \mathrm{U}$ mouse IFN- $\alpha$ daily on days 7 through 12 . For combination experiments, treatment schedules and cell doses were established for each tumor model to achieve $10 \%-20 \%$ tumor clearance by NDV or anti-PD-1/anti-PD-L1 as single agents. For experiments evaluating the combination therapy of NDV and anti-PD-1/anti-PD-L1 antibodies, B16-F10 tumors were implanted by intradermal (i.d.) injection of $4 \times 10^{5}$ B16-F10 cells into the right flank and $5 \times 10^{4}$ cells into the left flank on day 0 . The site of tumor cell injection was circled with an ethanol-proof marker to ease subsequent virus administration. On days $3,5,7$, and 9 , the mice were treated with intratumoral injections of $2 \times 10^{7} \mathrm{PFU}$ of NDV in PBS in a total volume of $100 \mu$ l. Concurrently, on days $3,5,7$, and 9 , the mice received i.p. injections of anti-PD-1 antibody $(250 \mu \mathrm{g})$ or anti-PD-L1 antibody $(250 \mu \mathrm{g})$. The control groups received a corresponding i.p. dose of isotype antibody and an intratumoral injection of PBS. This cell dose and treatment schedule were established to ensure complete rejection of the treated tumors in the majority of the animals and to allow the readout of the efficacy of the combination to be driven primarily by the distant tumors. The animals were euthanized for signs of distress or when the total tumor volume reached $1,000 \mathrm{~mm}^{3}$. For depletion of immune cells, mice were injected i.p. with $500 \mu \mathrm{g}$ monoclonal antibodies against $\mathrm{CD}^{+}, \mathrm{CD} 4^{+}$, or NK1.1 either the day before tumor implantation (day -1) or on the first day of treatment (day 0), followed by injection of $250 \mu$ g every 5 days throughout the experiment. For the CT26 model, $5 \times 10^{5}$ cells were implanted into the right and left flanks on day 0 . Treatment was administered on days 7, 9, 11, and 13 in a manner similar to that described above.

Bioluminescence imaging. Mice were imaged every 2 to 3 days starting on day 6. Mice were injected retro-orbitally with $50 \mu \mathrm{l}$ of $40 \mathrm{mg} /$ $\mathrm{ml}$ D-luciferin (Caliper Life Sciences) in PBS and imaged immediately using the IVIS Imaging System (Caliper Life Sciences). Grayscale photographic images and bioluminescence color images were superimposed using The Living Image, version 4.0, software overlay (Caliper Life Sciences). A region of interest (ROI) was manually selected over the tumor, and the area of the ROI was kept constant.

Isolation of TILs. B16-F10 tumors were implanted by i.d. injection of $4 \times 10^{5}$ B16-F10 cells into the right flank and $2 \times 10^{5}$ cells into the left flank on day 0 . On days 7, 9, and 11, the mice were treated with intratumoral injections of $2 \times 10^{7} \mathrm{PFU}$ of NDV and with i.p. anti-PD- 1 antibody where specified (Figure 6 legend). The rare animals that died from tumor burden (always in the untreated control groups) or the animals that completely cleared the tumors (always in the treatment groups) were not used for the analysis. On day 15 , the mice were sacrificed, and tumors, spleens, and tumor-draining lymph nodes were removed using forceps and surgical scissors and weighed. Tumors from each group were minced with scissors prior to incubation with 1.67 Wünsch $\mathrm{U} / \mathrm{ml}$ Liberase and $0.2 \mathrm{mg} / \mathrm{ml}$ DNase for 30 minutes at $37^{\circ} \mathrm{C}$. Tumors were homogenized by repeated pipetting and filtered through a 70- $\mu \mathrm{m}$ nylon filter. Cell suspensions were washed once with complete RPMI and processed for flow cytometry. Cells from tumor-draining lymph nodes were isolated by grinding the lymph nodes through a 70- $\mu$ m nylon filter.

Flow cytometry. Cells isolated from tumors or tumor-draining lymph nodes were processed for surface labeling. Fixable viability dye eFluor 506 (eBioscience) was used to distinguish the live cells. Cells were further permeabilized using a FoxP3 Fixation and Permeabilization Kit (eBioscience) and stained for Ki-67, FoxP3, GrB, and IFN- $\gamma$. Data were acquired using the LSRII Flow Cytometer (BD Biosciences) and analyzed with FlowJo software.

DC purification and loading. Spleens from naive mice were isolated and digested with 1.67 Wünsch $\mathrm{U} / \mathrm{ml}$ Liberase and $0.2 \mathrm{mg} / \mathrm{ml}$ DNase for 30 minutes at $37^{\circ} \mathrm{C}$. The resulting cell suspensions were filtered through a 70- $\mu \mathrm{m}$ nylon filter and washed once with complete RPMI. CD11 $\mathrm{c}^{+}$DCs were purified by positive selection using Miltenyi Biotec magnetic beads. Isolated DCs were cultured overnight with recombinant GM-CSF and B16-F10 or TRAMP C2 tumor lysates and were purified on a Ficoll gradient.

Analysis of cytokine production. Cell suspensions from tumors were pooled and enriched for $\mathrm{CD}^{+} \mathrm{T}$ cells using a Miltenyi Biotec CD8 T Cell Purification Kit. Isolated $\mathrm{T}$ cells were counted and cocultured for 8 hours with DCs loaded with B16-F10 or TRAMP C2 tumor cell lysates in the presence of $20 \mathrm{U} / \mathrm{ml} \mathrm{IL-2} \mathrm{(R \& D} \mathrm{Systems)} \mathrm{plus} \mathrm{brefeldin}$ A (BD Biosciences) at a DC/lymphocyte ratio of 1:5. After restimulation, lymphocytes were processed for flow cytometry as above. 
Immunofluorescence and microscopy. Tumors were dissected from the mice, washed in PBS, fixed in $4 \%$ paraformaldehyde, and processed for paraffin embedment according to protocols described previously (55). Sections were cut using a microtome, mounted onto slides, and processed for staining with $\mathrm{H} \& \mathrm{E}$ or with anti-CD3, antiCD11b, and anti-PD-L1 antibody (rabbit clone E1L3N, catalog 13684; Cell Signaling Technology; $1.67 \mu \mathrm{g} / \mathrm{ml}$ ), with an automated Leica Bond RX processor using a previously published protocol (55). Slides were digitized using a Panoramic Flash Scanner (3DHISTECH).

NanoString gene expression analyses. B16-F10 tumors were implanted by i.d. injection of $4 \times 10^{5}$ B16-F10 cells into the right flank on day 0 . On days 7,10 , and 13 , the mice were treated with 3 intratumoral injections of NDV $\left(2 \times 10^{7} \mathrm{PFU}\right)$. On day 15, the animals were euthanized, and the tumors were excised, placed in TRIzol Reagent (Invitrogen, Thermo Fisher Scientific), and homogenized. The samples were flash-frozen in dry ice and ethanol and stored at $-80^{\circ} \mathrm{C}$. RNA was later purified from TRIzol using the Direct-zol RNA MiniPrep Kit (Zymo Research). Isolated RNA was hybridized with the NanoString nCounter PanCancer Immune Profiling Mouse Panel CodeSet and quantified using the nCounter Digital Analyzer at the MSKCC Genomics Core Facility. Data were processed with nSolver Analysis Software (NanoString) using the Advanced Analysis module.

Extraction of RNA and real-time quantitative PCR. Mice were challenged with i.d. injection of $5 \times 10^{5}$ B16-F10 cells into the right flank and $1 \times 10^{5}$ B16-F10 cells into the left flank on day 0 . On days 7, 9, and 11 , mice were treated with 3 intratumoral injections of PBS or NDV $(2 \times$ $10^{7} \mathrm{PFU}$ in $100 \mu \mathrm{l}$ ) into the right-flank tumors and i.p. injections of PBS or $250 \mu \mathrm{g}$ anti-PD-1 antibody (clone RMP1-14). On day 14, the animals were euthanized, and the tumors were excised and snap-frozen in liquid nitrogen. The samples were homogenized, and DNA and RNA were extracted using the AllPrep DNA/RNA Mini Kit (QIAGEN) according to the manufacturer's instructions. The samples were stored at $-80^{\circ} \mathrm{C}$ until RT-PCR was performed with a High Capacity cDNA Reverse Transcription Kit (Applied Biosystems). No preamplification of cDNA products was performed. For real-time PCR, we used VeriQuest Probe One-Step qRT-PCR Master Mix (2X) (Affymetrix) and TaqMan Gene Expression Assays (Thermo Fisher Scientific) for each gene analyzed, as well Actb and GAPDH as housekeeping genes. Real-time PCR was performed according to the manufacturer's instructions using the Fluidigm Biomark HD platform on a 48.48 IFC chip with the provided reagents (Fluidigm user guide PN 68000089 H1). Real-time PCR results were analyzed using Real-Time PCR Analysis Software, version 4.3.1 (Fluidigm), relative gene expression was normalized to GAPDH, and data were exported to Excel. The heatmaps show a direct representation of the $\mathrm{Ct}$ values without further manipulation. Samples with no available data are shown in gray.

For analysis of gene expression from tumor histocultures, total RNA was isolated by homogenization of tissue into RNA STAT-60 (Tel-Test Inc.) using a polytron homogenizer and then total RNA was extracted according to the manufacturer's instructions. After isopropanol precipitation, total RNA was re-extracted with phenol/ chloroform/isoamyl alcohol (25:24:1) (Sigma-Aldrich) using phaselock light tubes (Thermo Fisher Scientific). DNase-treated total RNA was reverse transcribed using QuantiTect Reverse Transcription (QIAGEN) according to the manufacturer's protocol. Primers were obtained from Life Technologies (Thermo Fisher Scientific). Real-time quantitative PCR on 10 ng cDNA from each sample was performed using unlabeled primers at $900 \mathrm{nM}$ each with $250 \mathrm{nM}$ FAM-labeled probe in a TaqMan RT-qPCR reaction on the Fluidigm Biomark Sequence Detection System. Ubiquitin levels were measured in a separate reaction and were used to normalize the data by the $\Delta \Delta \mathrm{Ct}$ method. Using the mean $\mathrm{Ct}$ value for ubiquitin and the gene of interest for each sample, the following equation was applied to obtain the normalized values: $1.8^{\text {(Ct ubiquitin-Ct gene of interest) }} \times 10^{4}$.

Statistics. The primary research objective was to define and characterize the inhibitory pathways upregulated in the tumor microenvironment by NDV. The prespecified hypothesis suggested that NDV infection would induce the upregulation of compensatory inhibitory pathways, which could be further targeted using systemic strategies. The overall study design involved a series of controlled laboratory experiments in mice, as described in the sections below. In all of the studies, the assignment of animals to experimental groups was random. For survival studies, sample sizes of 5 to 10 mice per group were used, and pooled data from several replicate experiments were used for statistics. With 20 mice per group, $90 \%$ power, and a 5\% significance level, we could detect differences in tumor-free survival from $40 \%$ to $70 \%$. Survival analyses were performed using the log-rank test. The experiments were replicated 2-3 times as noted in the figure legends, and the final analysis included either pooled data or representative experiments. For the experiments reporting isolation of TILs, 5-10 mice per group were used for each experiment, with 2 to 5 replicates. All outliers were included in the data analysis. In the experiments focusing on the analysis of TILs, animals that died or animals that completely rejected the tumors were not included because of a lack of tissue for analysis. In all experiments, mice were randomly assigned to specific treatment groups by cage, with randomization performed on the basis of the cage position on the rack. Data were analyzed by 2-tailed Student's $t$ test (for comparisons of 2 groups) or ANOVA (for comparisons of $\geq 3$ groups), as appropriate. Survival data were analyzed using a log-rank (Mantel-Cox) test. A 2-sided $P$ value of less than 0.05 was considered statistically significant. For mouse RT-PCR gene expression analyses, the $Z$ score for the presented gene set was calculated using the following formula: $Z=(\mathrm{X}-\mu) / \sigma$, where $\mathrm{X}$ is the $\mathrm{Ct}$ value for a specific gene, $\mu$ is the mean of all values for the gene, and $\sigma$ is the standard deviation of all values for the gene. The mean $Z$ score $(\mu Z)$ for each sample was calculated as follows: $\mu Z=$ $\Sigma_{\text {(all genes) }} Z_{\text {sample }} /$ number of genes. Gene expression data and cytokine ELISA data from human histocultures were analyzed using a Wilcoxon matched-pairs, signed-rank test.

Study approval. All mouse procedures and experiments for this study were approved by the IACUC of the MSKCC. Mice were treated in accordance with NIH and American Association of Laboratory Animal Care regulations. Human tumor specimens were obtained in compliance with state and federal regulations from commercial sources (Folio, Bio-Options, and MT Group) or from the University of Rochester, in accordance with the tissue protocol approved by the IRB of the University of Rochester (Rochester, Minnesota, USA) and after obtaining written informed consent from the patients. Blood from patients with cancer was obtained from Conversant Bio, and normal blood came from the in-house healthy donor program at MRL.

\section{Author contributions}

DZ designed and performed the experiments, analyzed the data, and prepared the manuscript. JMR, AO, MG, SS, YY, JW, and WMB 
performed the experiments and assisted with data analysis and manuscript preparation. TM and JDW assisted with experimental design, data interpretation, and manuscript preparation.

\section{Acknowledgments}

This work was supported by the National Institutes of Health (CA056821 to JDW). The laboratory of JDW and TM is supported by the Ludwig Institute for Cancer Research and Swim Across America. DZ received funding from the Damon Runyon Cancer Research Foundation, Bladder Cancer Awareness Network, Ovar- ian Cancer Research Foundation, MSKCC Cycle for Survival, and the Department of Defense Ovarian Cancer Research Academy (OC150111). DZ, TM, and JDW are members of the Parker Institute for Cancer Immunotherapy, which supports the MSKCC Cancer Immunotherapy Program. MSKCC is supported by the NCI Core grant P30 CA008748.

Address correspondence to: Jedd Wolchok, Memorial Sloan-Kettering Cancer Center, 1275 York Avenue, Box 340, New York, New York 10065, USA. Phone: 646.888.2315; Email: wolchokj@mskcc.org.
1. Hotte SJ, et al. An optimized clinical regimen for the oncolytic virus PV701. Clin Cancer Res. 2007;13(3):977-985.

2. Kaufman HL, Kim DW, DeRaffele G, Mitcham J, Coffin RS, Kim-Schulze S. Local and distant immunity induced by intralesional vaccination with an oncolytic herpes virus encoding GM-CSF in patients with stage IIIc and IV melanoma. Ann Surg Oncol. 2010;17(3):718-730.

3. Andtbacka RH, et al. Talimogene laherparepvec improves durable response rate in patients with advanced melanoma. JClin Oncol. 2015;33(25):2780-2788

4. Zamarin D, Palese P. Oncolytic Newcastle disease virus for cancer therapy: old challenges and new directions. Future Microbiol. 2012;7(3):347-367.

5. Zamarin D, et al. Localized oncolytic virotherapy overcomes systemic tumor resistance to immune checkpoint blockade immunotherapy. Sci Transl Med. 2014;6(226):226ra32.

6. Zamarin D, et al. Intratumoral modulation of the inducible co-stimulator ICOS by recombinant oncolytic virus promotes systemic anti-tumour immunity. Nat Commun. 2017;8:14340.

7. Puzanov I, et al. Talimogene laherparepvec in combination with ipilimumab in previously untreated, unresectable stage IIIB-IV melanoma. JClin Oncol. 2016;34(22):2619-2626.

8. Ribas A, et al. Oncolytic virotherapy promotes intratumoral $\mathrm{T}$ cell infiltration and improves anti-PD-1 immunotherapy. Cell. 2017;170(6):1109-1119.e10.

9. Chesney J, et al. Randomized, open-label phase II study evaluating the efficacy safety of Talimogene laherparepvec in combination with ipilimumab versus ipilimumab alone in patients with advanced, unresectable melanoma [published online ahead of print October 5, 2017]. J Clin Oncol. https://doi.org/10.1200/JCO.2017.73.7379.

10. Zitvogel L, Galluzzi L, Kepp O, Smyth MJ, Kroemer G. Type I interferons in anticancer immunity. Nat Rev Immunol. 2015;15(7):405-414.

11. Fuertes MB, et al. Host type I IFN signals are required for antitumor $\mathrm{CD} 8^{+} \mathrm{T}$ cell responses through $\mathrm{CD} 8 \alpha^{+}$dendritic cells. JExp Med. 2011;208(10):2005-2016.

12. Diamond MS, et al. Type I interferon is selectively required by dendritic cells for immune rejection of tumors. J Exp Med. 2011;208(10):1989-2003.

13. Kato H, et al. Cell type-specific involvement of RIG-I in antiviral response. Immunity. 2005;23(1):19-28.

14. Ayers $\mathrm{M}$, et al. IFN- $\gamma$-related mRNA profile predicts clinical response to PD-1 blockade. JClin
Invest. 2017;127(8):2930-2940.

15. Hugo W, et al. Genomic and transcriptomic features of response to anti-PD-1 therapy in metastatic melanoma. Cell. 2016;165(1):35-44.

16. Shalapour S, et al. Immunosuppressive plasma cells impede T-cell-dependent immunogenic chemotherapy. Nature. 2015;521(7550):94-98.

17. Ammirante M, et al. An IKK $\alpha$-E2F1-BMI1 cascade activated by infiltrating $\mathrm{B}$ cells controls prostate regeneration and tumor recurrence. Genes Dev. 2013;27(13):1435-1440.

18. Ammirante M, Luo JL, Grivennikov S, Nedospasov S, Karin M. B-cell-derived lymphotoxin promotes castration-resistant prostate cancer. Nature. 2010;464(7286):302-305.

19. Sharpe AH, Wherry EJ, Ahmed R, Freeman GJ. The function of programmed cell death 1 and its ligands in regulating autoimmunity and infection. Nat Immunol. 2007;8(3):239-245.

20. Blackburn SD, et al. Coregulation of $\mathrm{CD}^{+} \mathrm{T}$ cell exhaustion by multiple inhibitory receptors during chronic viral infection. Nat Immunol. 2009;10(1):29-37.

21. Paley MA, et al. Progenitor and terminal subsets of $\mathrm{CD}^{+} \mathrm{T}$ cells cooperate to contain chronic vira infection. Science. 2012;338(6111):1220-1225.

22. Spranger S, et al. Up-regulation of PD-L1, IDO, and $\mathrm{T}$ (regs) in the melanoma tumor microenvironment is driven by CD8(+) T cells. Sci Transl Med. 2013;5(200):200ra116.

23. Tumeh PC, et al. PD-1 blockade induces respons es by inhibiting adaptive immune resistance. Nature. 2014;515(7528):568-571.

24. Taube JM, et al. Colocalization of inflammatory response with B7-h1 expression in human melanocytic lesions supports an adaptive resistance mechanism of immune escape. Sci Transl Med. 2012;4(127):127ra37.

25. Dankort D, et al. Braf(V600E) cooperates with Pten loss to induce metastatic melanoma. Nat Genet. 2009;41(5):544-552.

26. Charlton JJ, Tsoukatou D, Mamalaki C, Chatzidakis I. Programmed death 1 regulates memory phenotype CD4 T cell accumulation, inhibits expansion of the effector memory phenotype subset and modulates production of effector cytokines. PLoS One. 2015;10(3):e0119200.

27. Charlton JJ, Chatzidakis I, Tsoukatou D, Boumpas DT, Garinis GA, Mamalaki C. Programmed death- 1 shapes memory phenotype CD8 T cell subsets in a cell-intrinsic manner. J Immunol. 2013;190(12):6104-6114

28. Jarahian M, et al. Activation of natural killer cells by Newcastle disease virus hemagglutinin- neuraminidase. J Virol. 2009;83(16):8108-8121.

29. Zamarin D, Wolchok JD. Potentiation of immunomodulatory antibody therapy with oncolytic viruses for treatment of cancer. Mol Ther Oncolytics. 2014;1:14004.

30. Lichty BD, Breitbach CJ, Stojdl DF, Bell JC. Going viral with cancer immunotherapy. Nat Rev Cancer. 2014;14(8):559-567.

31. Galluzzi L, Buqué A, Kepp O, Zitvogel L, Kroemer G. Immunogenic cell death in cancer and infectious disease. Nat Rev Immunol. 2017;17(2):97-111.

32. Guo ZS, Liu Z, Bartlett DL. Oncolytic immunotherapy: dying the right way is a key to eliciting potent antitumor immunity. Front Oncol. 2014;4:74.

33. Wong J, Schulman A, Kelly K, Zamarin D, Palese P, Fong Y. Detection of free peritoneal cancer cells in gastric cancer using cancer-specific Newcastle disease virus. J Gastrointest Surg. 2010;14(1):7-14.

34. Song KY, Wong J, Gonzalez L, Sheng G, Zamarin D, Fong Y. Antitumor efficacy of viral therapy using genetically engineered Newcastle disease virus [NDV(F3aa)-GFP] for peritoneally disseminated gastric cancer. JMol Med. 2010;88(6):589-596.

35. Silberhumer GR, et al. Genetically engineered oncolytic Newcastle disease virus effectively induces sustained remission of malignant pleural mesothelioma. Mol Cancer Ther. 2010;9(10):2761-2769.

36. Li P, et al. Therapeutic effects of a fusogenic Newcastle disease virus in treating head and neck cancer. Head Neck. 2011;33(10):1394-1399.

37. Zamarin D, Vigil A, Kelly K, García-Sastre A, Fong Y. Genetically engineered Newcastle disease virus for malignant melanoma therapy. Gene Ther. 2009;16(6):796-804.

38. Lorence RM, et al. Phase 1 clinical experience using intravenous administration of PV701, an oncolytic Newcastle disease virus. Curr Cancer Drug Targets. 2007;7(2):157-167.

39. Holmgaard RB, Zamarin D, Munn DH, Wolchok JD, Allison JP. Indoleamine 2,3-dioxygenase is a critical resistance mechanism in antitumor $\mathrm{T}$ cell immunotherapy targeting CTLA-4. J Exp Med. 2013;210(7):1389-1402.

40. Topalian SL, et al. Safety, activity, and immune correlates of anti-PD-1 antibody in cancer. $N$ Engl JMed. 2012;366(26):2443-2454.

41. Rosenberg JE, et al. Atezolizumab in patients with locally advanced and metastatic urothelial carcinoma who have progressed following treatment with platinum-based chemotherapy: a single-arm, multicentre, phase 2 trial. Lancet. 2016;387(10031):1909-1920.

42. Pardoll DM. The blockade of immune check- 
points in cancer immunotherapy. Nat Rev Cancer. 2012;12(4):252-264.

43. Kaufman HL, et al. Interim results of the CAPRA clinical trial: CAVATAK and pemrolizumab in advanced melanoma. JITC. 2016;4(suppl 1):73.

44. Liu Z, Ravindranathan R, Kalinski P, Guo ZS, Bartlett DL. Rational combination of oncolytic vaccinia virus and PD-L1 blockade works synergistically to enhance therapeutic efficacy. Nat Commun. 2017;8:14754.

45. Ilett E, et al. Prime-boost using separate oncolytic viruses in combination with checkpoint blockade improves anti-tumour therapy. Gene Ther. 2017;24(1):21-30.

46. Hardcastle J, et al. Immunovirotherapy with measles virus strains in combination with anti-PD-1 antibody blockade enhances antitumor activity in glioblastoma treatment. Neuro-oncology. 2017;19(4):493-502.
47. Rajani K, et al. Combination therapy with reovirus and anti-PD-1 blockade controls tumor growth through innate and adaptive immune responses. Mol Ther. 2016;24(1):166-174.

48. Chen CY, et al. Cooperation of oncolytic herpes virotherapy and pd-1 blockade in murine rhabdomyosarcoma models. Sci Rep. 2017;7(1):2396.

49. Saha D, Martuza RL, Rabkin SD. Macrophage polarization contributes to glioblastoma eradication by combination immunovirotherapy and immune checkpoint blockade. Cancer Cell. 2017;32(2):253-267.e5.

50. Shim KG, et al. Inhibitory receptors induced by VSV viroimmunotherapy are not necessarily targets for improving treatment efficacy. Mol Ther. 2017;25(4):962-975.

51. Streby KA, et al. Intratumoral injection of HSV1716, an oncolytic herpes virus, is safe and shows evidence of immune response and viral replication in young cancer patients. Clin Cancer Res. 2017;23(14):3566-3574.

52. Rashidian M, et al. Predicting the response to CTLA- 4 blockade by longitudinal noninvasive monitoring of CD8 T cells. J Exp Med. 2017;214(8):2243-2255.

53. Ayllon J, Garcia-Sastre A, Martinez-Sobrido L. Rescue of recombinant Newcastle disease virus from cDNA.J Vis Exp. 2013;(80):50830.

54. Sheehan KC, et al. Blocking monoclonal antibodies specific for mouse IFN-alpha/beta receptor subunit 1 (IFNAR-1) from mice immunized by in vivo hydrodynamic transfection. J Interferon Cytokine Res. 2006;26(11):804-819.

55. Ricca J, et al. Validation of anti-mouse PDL-1 goat polyclonal antibody staining with mouse PDL-1 in situ hybridization on adjacent Sections of cell pellets and mouse tumors. Methods Mol Biol. 2017;1554:253-262. 